CERN LIBRARIES, GENEVA

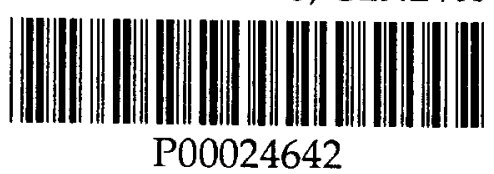

\title{
Test of Internal Halo Targets in the HERA Proton Ring
}

\author{
C. Hast \\ Institut für Physik, Universität Dortmund \\ K. Ehret, W. Hofmann, S. Khan, K. T. Knöpfle, M. Reber \\ J. Rieling, M. Spahn, J. Spengler, T. Lohse \\ Max-Planck-Institut für Kernphysik, Heidelberg \\ V. Pugatch \\ Institute for Nuclear Research, Ukrainian Academy of Science, Kiev, Ukraine
}





\title{
Test of Internal Halo Targets in the HERA Proton Ring
}

\author{
C. Hast \\ Institut für Physik, Universität Dortmund, Germany \\ K. Ehret, W. Hofmann, S. Khan ${ }^{1}$, K.T. Knöpfle, M. Reber, \\ J. Rieling, M. Spahn, J. Spengler, T. Lohse ${ }^{2}$ \\ Max-Planck-Institut für Kernphysik, Heidelberg, Germany \\ V. Pugatch \\ Institute for Nuclear Research, Ckrainian Academy of Science. Kiev, Ukraine
}

Submitted to

Nucl. Inst. and Meth. A

\begin{abstract}
Internal wire targets in the halo of stored proton beams provide a line source of protonnucleus interactions for highest-rate fixed target experiments. We have studied such internal halo targets at the $820 \mathrm{GeV}$ proton ring of the HERA ep collider. The tests showed that most of the protons in the beam halo - which would otherwise hit the collimators - can be brought to interaction in a relatively thin target wire at distances of 7 to 8 beam widths from the center of the beam. At less than $10 \%$ of the HERA total design current, and less than $20 \%$ of the current per bunch, interaction rates up to $8 \mathrm{MHz}$ were observed, corresponding to more than 2 interactions per bunch crossing. The halo targets were used in parallel to the HERA luminosity operation; no significant disturbances of the HERA ep experiments, of the machine stability or beam quality were observed. We present data on the steady-state and transient behaviour of interaction rates and discuss the interpretation in terms of a simple beam dynamics model. Issues of short-, medium- and long-term rate fluctuations and of rate stabilization by feedback are addressed.
\end{abstract}

\footnotetext{
${ }^{1}$ now at BESSY II, Berlin, Germany

${ }^{2}$ now at Institut für Physik, Humboldt Universität zu Berlin, Germany
} 


\section{Introduction}

One of the outstanding problems in High Energy Physics is the origin of CP violation, a phenomenon discovered 30 years ago [1] in decays of neutral kaons. A decisive test of the implementation of C.P violation in the standard model of electroweak interactions [2] requires the discovery anc accurate measurement of CP violation phenomena in systems heavier than kaons. The most promising laboratory for CP violation studies are decays of neutral $B$ mesons, where $\mathrm{CP}$ violating effects are expected to be large [3]. However, the decay channels which can exhibit CP asymmetries are extremely rare, typically suppressed by four to five orders of magnitude. Experimental cuts to select clean signatures and to identify the $B$ flavors reduce the useful rates further. An experiment will therefore require the production of very large numbers of $B$ mesons, i.e. a machine acting as a $B$ factory.

The most popular $B$ factory is a high luminosity $\mathrm{e}^{+} \mathrm{e}^{-}$collider with a center of mass energy corresponding to the mass of the $\Upsilon(4 \mathrm{~S})$ bottomium resonance which can decay into two neutral $B$ mesons. The appealing features of this approach are the simplicity of the events, the clean signatures and the wealth of heavy flavor physics accessible. In order to arrive at a measurement of CP violating effects, however, the storage rings have to be operated at up to now unreached luminosities. Ambitious projects have been started in Japan (KEK) [4] and the U.S.A. (SLAC) [5].

The second possibility to produce large numbers of $B$ mesons is offered by hadronic interactions at high energies. In this case, cross sections and therefore the rate of $B$ events are much higher than at $\mathrm{e}^{+} \mathrm{e}^{-}$machines; the events contain, however. a large number of particles besides the decay products of $B$ mesons and the background of events with no $B$ mesons produced is severe. This shifts the experimental challenge to the construction of adequate detectors and trigger systems. With increasing CM energy, the $B$ cross section in hadronic interactions rises and the fraction of non- $B$ background decreases, so large center of mass energies are of advantage.

In reference [6] the feasibility of using the existing HERA proton ring for a $B$ experiment is discussed. In a fixed target environment, the $820 \mathrm{GeV}$ proton beam energy leads to a center of mass energy $\sqrt{s} \approx 40 \mathrm{GeV}$, an energy not far above the $B$ threshold. At this relatively low energy the background of normal inelastic interactions dominates $B$ production by six orders of magnitude. A CP experiment therefore requires extreme event rates in the order of 30 to $50 \mathrm{MHz}$ during a running period of several years. Since the maximal bunch crossing (BX) frequency of the HERA proton ring amounts to $10 \mathrm{MHz}$, this means that several events must be produced per BX.

It has been proposed in [6] to use thin wires or ribbons as internal targets to achieve this goal. Such targets have been discussed theoretically in [7] and tested in low energy beams in [8]. At the $820 \mathrm{GeV}$ HERA proton ring, the targets are positioned around the proton beam at a distance of $4 \ldots 8$ r.m.s. beam widths, i.e. inside the beam halo but outside the beam core. The beam halo scraped by the target wires is continuously replenished by protons diffusing outward, a process mainly driven by the non-linear effects of ep beambeam interaction. Such a strategy should assure that the beam quality is not affected and the ep luminosity of the other HERA experiments is not reduced. It has to be verified, however, that most of the halo protons can be brought to interaction in the target before these particles (which have unstable orbits) are lost from the ring due to their outward drift 


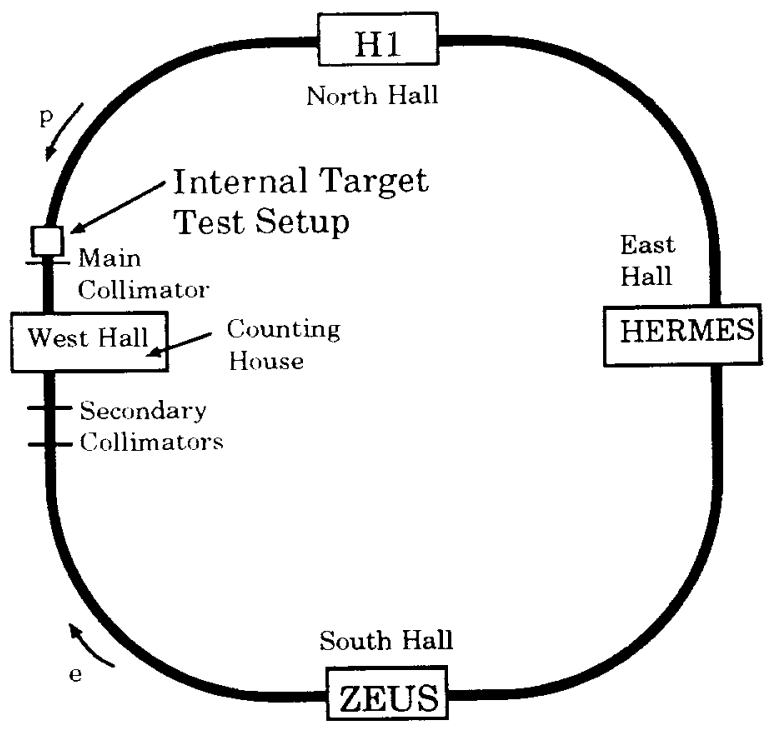

Figure 1: Sketch of the HERA rings with the location of the four experimental areas. Also shown is the position of the internal target test experiment close to the west hall.

in phase space (this happens on the time scale of seconds). For the nominal HERA beam parameters, the event rates of up to $50 \mathrm{MHz}$ can only be reached, if at least $50 \%$ of the halo protons are absorbed on the target.

In this article we report on test measurements performed with halo targets in the HERA proton ring during the running periods in 1992 and 1993. After a description of the relevant accelerator parameters in section 2 and of the experimental set-up in section 3 , we describe in section 4 the simulations which guided the design of the target and which allow to interpret the measurements in the framework of a simple beam dynamics model. The experimental results on interaction rates. transients, event topology and rate fluctuations are presented in sections 5-8. First experiences concerning automatic target control are summarized in section 9. The topic of compatibility with the normal machine operation is addressed in section 10 . The article concludes with a summary and outlook.

\section{The HERA Machine}

HERA is a double storage ring designed for colliding a $820 \mathrm{GeV}$ proton beam with a $30 \mathrm{GeV}$ electron beam. The rings have a length of $6335.8 \mathrm{~m}$. They are served by a complicated chain of injectors and preaccelerators, consisting of two injection LINACs, an electron accumulator, the upgraded DESY synchrotrons and the former $\mathrm{e}^{+} e^{-}$storage ring PETRA. HERA has four interaction regions as shown in Fig. 1. Two of them (north and south hall) house the general purpose ep detectors $\mathrm{H} 1$ and ZEUS. In the other two the beams are not brought to collision. The HERMES detector, using exclusively the electron beam, will be installed in the east hall. The west hall accommodates machine utilities and would offer space for a fourth experiment, such as a $B$ experiment (which would exclusively use the proton beam). The present experiment to test internal targets is located $118 \mathrm{~m}$ upstream of the HERA west hall, 
close to the aperture defining main collimators. Relevant parameters of the proton ring, as operated in the $1993 \mathrm{rm}$. are listed in table 1.

Table 1: Typical parameters of the proton beam during the 1.993 tests. All non-global parameters are given for the test location $118 \mathrm{~m}$ upstream of the HERA west hall. The horizontal direction is denoted by the index $x$, the vertical by $y$.

\begin{tabular}{|c|c|c|}
\hline \multicolumn{3}{|c|}{ Proton Beam: } \\
\hline Energy & \multicolumn{2}{|c|}{$820 \mathrm{GeV}$} \\
\hline Beta function & $\beta_{x}=94 \mathrm{~m}$ & $\beta_{y}=30 \mathrm{~m}$ \\
\hline Alpha & $\alpha_{x}=-1.68$ & $\alpha_{y}=1.10$ \\
\hline Typical emittance & $\epsilon_{x}=5 \ldots 6 \cdot 10^{-9} \mathrm{rad} \mathrm{m}$ & $\epsilon_{y}=4 \ldots 5 \cdot 10^{-9} \mathrm{rad} \mathrm{m}$ \\
\hline Emittance growth rate & $\approx 2.5 \cdot 10^{-10} \mathrm{rad} \mathrm{m} \mathrm{h}^{-1}$ & $\approx 1.5 \cdot 10^{-10} \mathrm{rad} \mathrm{m} \mathrm{h}^{-1}$ \\
\hline Spatial dispersion & $d_{x}=0.82 \mathrm{~m}$ & $d_{y}=0$ \\
\hline Angular dispersion & $d_{x^{\prime}}=24 \mathrm{mrad}$ & $d_{y^{\prime}}=0$ \\
\hline Beam size (r.m.s.) & $\sigma_{x}=0.70 \ldots 0.75 \mathrm{~mm}$ & $\sigma_{y}=0.35 \ldots 0.40 \mathrm{~mm}$ \\
\hline Beam divergence (r.m.s.) & $\sigma_{x^{\prime}}=14 \ldots 16 \mu \mathrm{rad}$ & $\sigma_{y^{\prime}}=17 \ldots 19 \mu \mathrm{rad}$ \\
\hline Typical tunes & $Q_{x}=31.291$ & $Q_{y}=31.294$ \\
\hline main collimator aperture & $8-12 \sigma_{x}$ & $8-12 \sigma_{y}$ \\
\hline revolution time & \multicolumn{2}{|c|}{$21.1 \mu \mathrm{s}$} \\
\hline $\mathrm{p}$ bunch trains & \multicolumn{2}{|c|}{9 (with 1440 ns spacing) } \\
\hline $\mathrm{p}$ bunches per train & \multicolumn{2}{|c|}{10 (with 96 ns spacing) } \\
\hline $\mathrm{p}$ bunches in collision & \multicolumn{2}{|c|}{84} \\
\hline protons per bunch & \multicolumn{2}{|c|}{$1.8 \cdot 10^{10}$} \\
\hline bunch length & \multicolumn{2}{|c|}{$1.3 \mathrm{~ns}$} \\
\hline Typical current & \multicolumn{2}{|c|}{$13 \mathrm{~mA}\left(\approx 1.7 \cdot 10^{12}\right.$ protons $)$} \\
\hline Range of beam lifetimes & \multicolumn{2}{|c|}{$20-500 \mathrm{~h}$} \\
\hline
\end{tabular}

The proton ring is equipped with $4.7 \mathrm{~T}$ superconducting bending magnets which allow a beam energy of $820 \mathrm{GeV}$. The machine is operated routinely at its nominal beam energy since its first year of operation (1992). The electron ring is designed for a beam energy of $30 \mathrm{GeV}$ but was operated in 1993 at $26 \mathrm{GeV}$.

Both rings operated with low beam intensities in 1993. In the electron ring, the intensity was limited by lifetime problems. The bunch intensity of the proton beam was limited in the injection chain. The total proton current reached typically $7-9 \%$ of the design current of $160 \mathrm{~mA} ; 90$ out of a total of 210 bunches were filled. The mean current per bunch was about $17 \%$ of the design value.

The bunches were organized in 9 trains, each consisting of 10 bunches. The bunch spacing within a train corresponded to $96 \mathrm{~ns}$; the spacing between two consecutive trains corresponded to $1344 \mathrm{~ns}$. The electron ring was filled such that the last six proton bunches in the last bunch train did not collide with electron bunches. These six bunches did not contribute significantly to the observed interaction rates since they were accompanied by a halo which was much smaller than that of the 84 colliding bunches.

We note that the emittance of the proton beam was about a factor of two below the design 


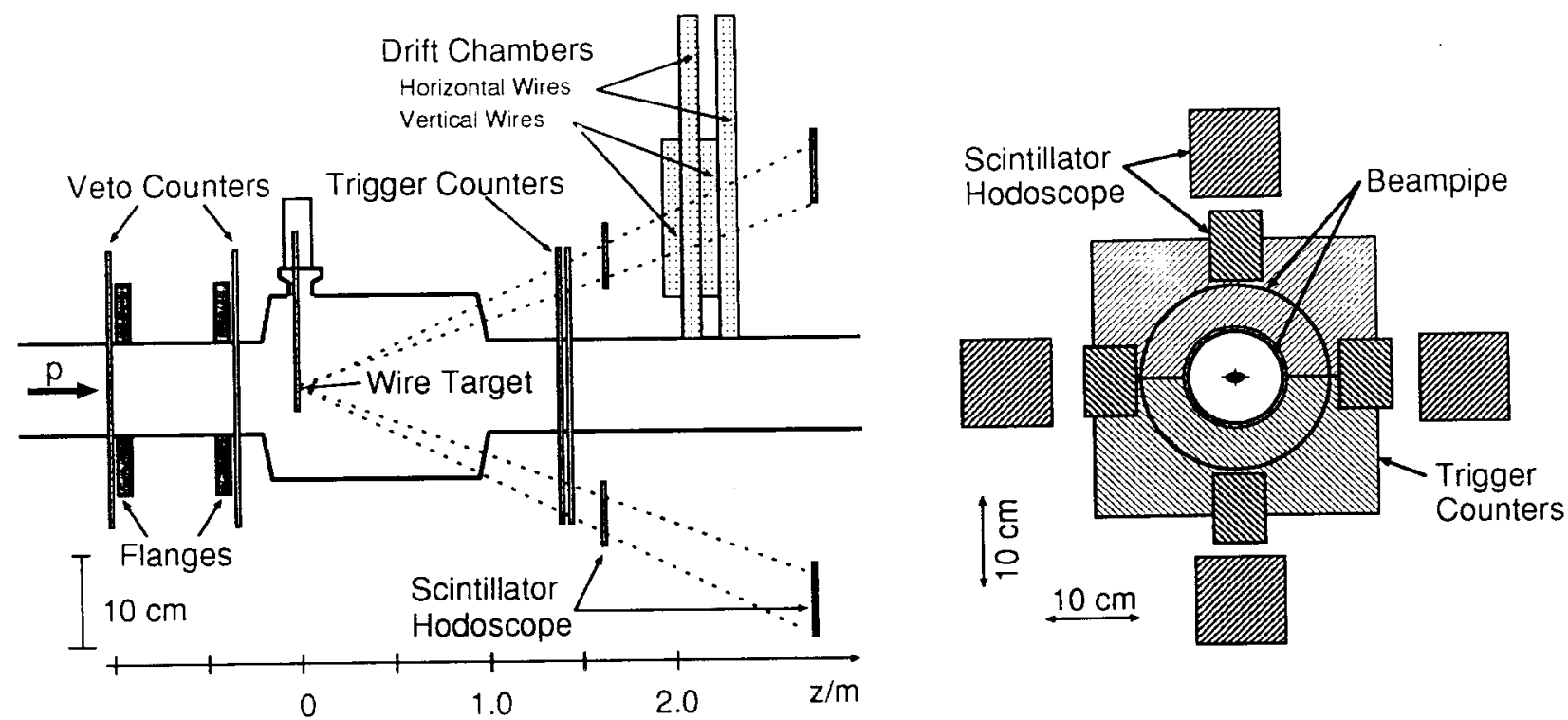

Figure 2: Sketch of the experimental set-up for the internal target tests (side and front view).

values. This compensated partly for the ep luminosity loss due to low beam intensities. The emittance was measured at the begin and end of each fill. When in the following the distance between a target wire and the beam is quoted in units of the beam widths, the actual measured emittance (which varied by $\approx 20 \%$ from run to run) is used rather than the nominal emittance.

The maximum luminosity achieved in 1993 amounted to $1.6 \cdot 10^{30} \mathrm{~cm}^{-2} \mathrm{~s}^{-1}$ which is $10 \%$ of the design value. An integrated luminosity of $1088 \mathrm{pb}^{-1}$ has been accumulated.

The luminosity lifetime amounted on average to $6 \mathrm{~h}$; it is dominated by the electron beam lifetime (typically $10 \mathrm{~h}$ ). The proton beam currents decay with much longer time constants, often larger than $100 \mathrm{~h}$. The useful storage time of the proton beam is, however, not governed by the current lifetime, but by a slow growth of the transverse beam emittance of $1 \ldots 3 \cdot 10^{-10} \mathrm{rad} \mathrm{m} \mathrm{h}^{-1}$ during ep collision. The proton beam is typically aborted and re-filled once per day. The proton injection and acceleration procedure took about $4 \mathrm{~h}$ on average in 1993 .

About $50 \%$ of the year 1993 was scheduled for luminosity operation. About one third of the time was needed for injection, acceleration and tuning procedures, about one third for recovery from technical faults and about one third was spent in luminosity operation.

\section{$3 \quad$ Experimental Set-up}

The experimental set-up is sketched in Fig. 2. The internal target is located $118 \mathrm{~m}$ upstream of the center of the HERA west hall close to the main collimator system. It consists of two movable target forks (see Fig. 3), one moving horizontally and one vertically. Each fork is equipped with one Aluminum and one Copper target ribbon, the dimensions and properties of which are listed in table 2 . The forks are aligned such that the proton beam 


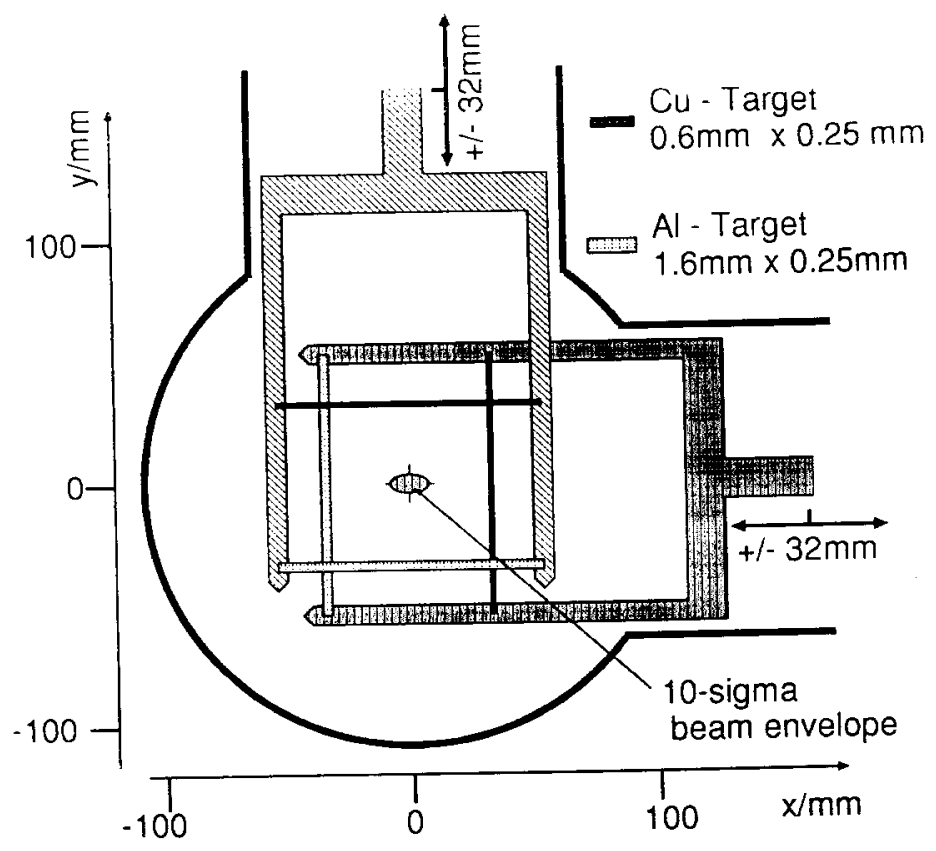

Figure 3: Arrangement of target ribbons on the movable target forks.

passes between the $\mathrm{Al}$ and $\mathrm{Cu}$ targets. Depending on the direction a fork is moved, either of the two targets on each fork can be driven into the beam halo, such that either a single horizontal or vertical ribbon or one horizontal and simultaneously one vertical ribbon can be positioned in the halo. For the $B$ experiment proposed in [6], multiple sets of target wires would be used, approaching the beam simultaneously from all sides. The total mass of those wires will be roughly equivalent to a single wire in the present test.

Table 2: Properties of the target ribbons used for the 1993 tests.

\begin{tabular}{|l|c|c|}
\hline \multicolumn{3}{|c|}{ Target Ribbons: } \\
\hline \hline Material & $\mathrm{Al}$ & $\mathrm{Cu}$ \\
\hline width along beam & $1.6 \mathrm{~mm}$ & $0.6 \mathrm{~mm}$ \\
& $0.4 \% \lambda_{I}$ & $0.4 \% \lambda_{I}$ \\
& $1.8 \% X_{0}$ & $4.2 \% X_{0}$ \\
\hline transverse width & $0.25 \mathrm{~mm}$ & $0.25 \mathrm{~mm}$ \\
\hline Interaction length $\lambda_{I}$ & $39.4 \mathrm{~cm}$ & $15.1 \mathrm{~cm}$ \\
\hline Radiation length $X_{0}$ & $8.89 \mathrm{~cm}$ & $1.43 \mathrm{~cm}$ \\
\hline Position (horizontal ribbon) & below beam & above beam \\
\hline Position (vertical ribbon) & inside & outside \\
\hline
\end{tabular}

The interaction trigger is provided by plastic scintillators close to the beam pipe, about $140 \mathrm{~cm}$ downstream of the target. The counters consist of $1 \mathrm{~cm} \mathrm{SCSN38} \mathrm{scintillator} \mathrm{and}$ have an area of $30 \times 30 \mathrm{~cm}^{2}$, with a central hole of $12 \mathrm{~cm}$ diameter for the beam pipe. For interactions at the target, they cover the range of polar angles between $40 \mathrm{mrad}$ and $110 \mathrm{mrad}$ 
corresponding to rapidities between 2.9 and 3.9 in the lab system. The $30 \times 30 \mathrm{~cm}^{2}$ counter is split into an upper and lower halve, each coupled to a wavelength shifter bar and read ont by a VALVO XP2011/03 photomultiplier. Two such systems are mounted behind each other. A coincidence between the signals from the upper or lower scintillators provides the trigger signal, with an efficiєncy well above $90 \%$ for inelastic $\mathrm{pN}$ interactions. Similarly, two sets of scintillators (VETO counters) are located $34 \mathrm{~cm}$ and $100 \mathrm{~cm}$ upstream of the target. Their purpose is to detect background events originating from protons lost upstream and to give timing information in order to detect backwards moving particles from interactions in the target.

Some information on the event topology is gained from four small solid angle scintillator hodoscopes. each consisting of two plastic scintillators $\left(53 \times 75 \mathrm{~mm}^{2}\right.$ and $\left.89 \times 89 \mathrm{~mm}^{2}\right)$. Their total acceptance for detection of interactions in the target was measured to be $\approx 60 \%$.

Tracking is provided by four small drift chambers, each consisting of 4 layers with 8 wires. The quadratic drift cells are defined by 14 field wires of $100 \mu \mathrm{m}$ diameter, surrounding the central $25 \mu \mathrm{m}$ sense wire which was operated at $3050 \mathrm{~V}$ (using $C F_{4}$ as a drift gas). The chambers provide typical point resolutions of $500 \mu \mathrm{m}$. The tracking chambers have an active area of $17 \times 8 \mathrm{~cm}^{2}$, which overlaps with the acceptance of one of the scintillator hodoscopes. They are arranged such that the wire orientation alternates from chamber to chamber between horizontal and vertical direction. The purpose of the drift chambers is to verify that single tracks from interactions are really originating in the target. More details on the calibration and operation of the chambers can be found in [9].

The beam position is monitored by close-by HERA beam pick-ups. Their information is useful for measuring relative beam positions. The absolute distance between target and beam can be inferred indirectly: First, the (nearby) main collimator jaws are moved towards the beam from left and right (top and bottom) until the beam is scraped equally on both sides (this can be performed with the help of loss detectors close to the collimators). From the collimator positions the beam position can then be computed in the collimator coordinate system. This yields a transformation between beam pick-up and collimator coordinates. Secondly, the target position can be absolutely calibrated in the collimator coordinate system. using the fact that interaction rates rise sharply when the target is moved out of the collimator shadow.

The data acquisition system is sketched in Fig. 4. The photomultiplier signals are amplified and shaped, and are fed into both discriminators and charge-sensitive ADCs. The $\mathrm{BX}$ signal of the first bunch (given by the central HERA bunch clock, derived from the RF system) starts TDCs for all scintillators and provides the gates for the ADCs. The TDCs receive the stop signals from the discriminators (or run into overflow if the signals are below threshold). Trigger signals are derived exclusively for the first BX in order to avoid pile-up from preceding bunches. In parallel all scintillator signals are fed into a $40 \mathrm{MHz}$ Flash-ADC system which digitizes the full length of a bunch train. This provides additional information on the time structure of the signals and on the structure of events in bunches following the leading bunch [10]. The Flash-ADCs are read out by a Motorola 68040 based VME microprocessor. Finally. the wire signals from the drift chambers are fed into a TDC system which measures drift-times for individual wires. The whole information of an event, containing the $A D C$ and TDC readings for all scintillators, the Flash-ADC memories as sent by the VME system, and the drift-time measurements from the chambers are read via CAMAC by a 


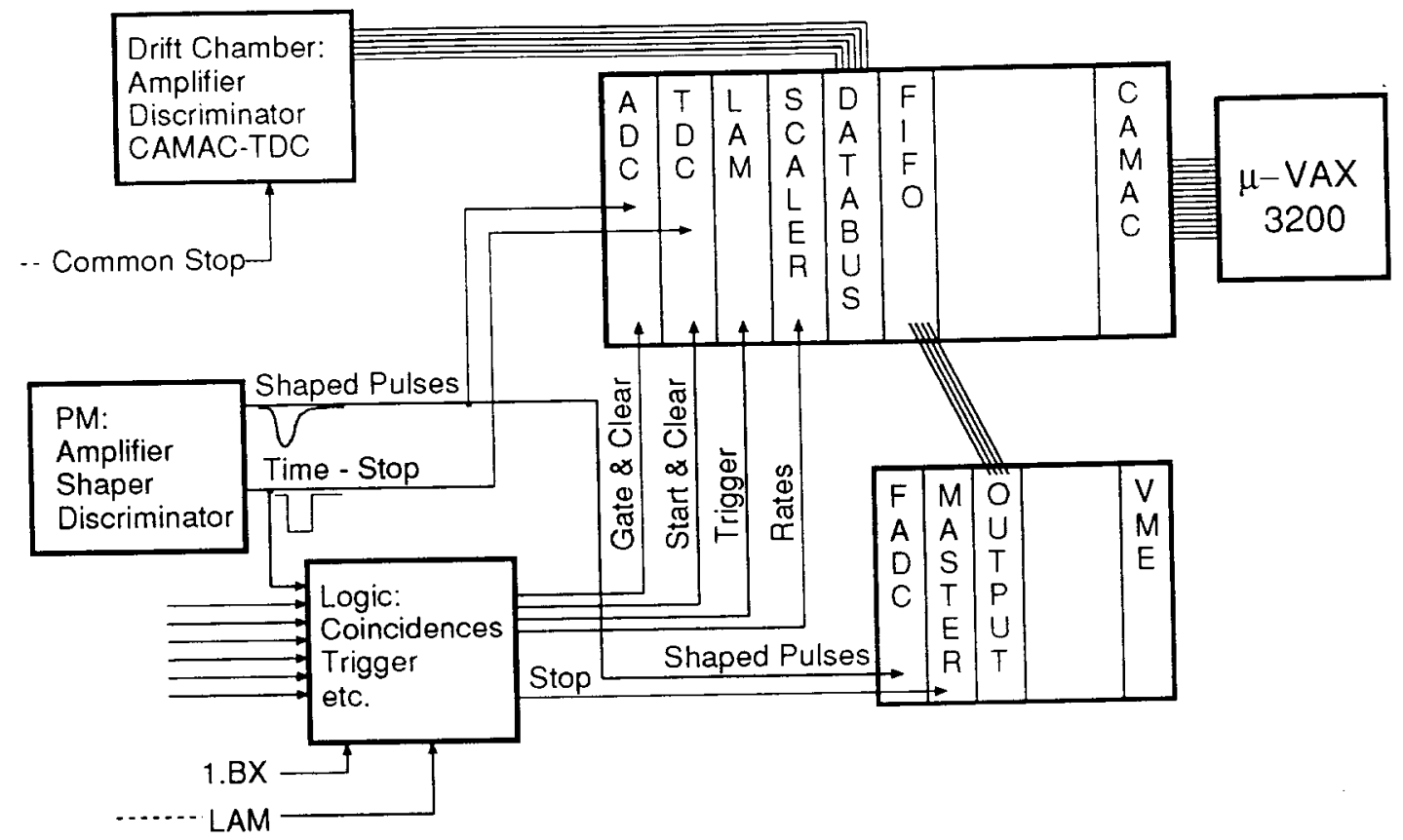

Figure 4: Sketch of the data acquisition system of the test experiment.

$\mu$ VAX3200 and written to tape.

Since the readout on an event by event basis suffers from significant deadtime, all relevant logical combinations of scintillator signals are fed into scalers for all bunch crossings, and these dead-time free counting rates are independently recorded every second. Machine parameters like the proton current, the beam lifetime, the beam pick-up signals, and the loss rates on the collimator jaws are also recorded in time intervals between 10 seconds and one minute.

Background information from ZEUS and $\mathrm{H} 1$ is continuously displayed in the control room and archived by the experiments.

\section{Target Simulations}

\subsection{General Framework}

Simulation of the interaction of the beam halo and the target is a vital tool for the optimization of the target arrangement. It also provides a framework for a quantitative interpretation of the observed interaction rates and at the same time reveals some properties of the beam halo. The latter is an important aspect, since the phase space region of the extreme halo is expected to be populated by a complicated mixture of beam particles on quasi-stable, on diverging, and on chaotic trajectories. The phase space structure and the beam dynamics depend critically on the machine set-up and on imperfections; in particular it is sensitive to the strength of betatron resonances of very high order [11]. The understanding of the drnamics of the extreme halo is therefore a delicate theoretical challenge.

Our simulations are based on highly simplified models, in which the complicated dynamics 
of the beam halo is globally parameterized as a diffusion process. Such models turned out to be successful in describing the loss rates monitored by pin-diodes in the vicinity of the main collimators after movements of the collimator jaws [12]. The dynamics of the halo particles is in this picture governed by the following processes:

1. Diffusion: The halo is continuously populated from the beam core by diffusion-like processes. The diffusion constants are expected to increase with a high power of the betatron amplitude [11]. In the extreme halo (around 8 to 10 r.m.s. beam widths distance from the core) the diffusion becomes very large and particles are lost on the time-scale of seconds or even fractions of a second on the aperture limitations (collimators). The diffusion process is regarded as an effective description of the superposition and interplay of all non-linear effects in the machine. This includes non-linear optics components from imperfections, beam-gas scattering, power supply ripple, and most importantly the beam-beam interaction force and its coupling to high order betatron resonances [11]. The diffusion is in this picture the only source of the finite currentand emittance-lifetimes.

\section{Target Hits:}

Once a halo particle has reached betatron amplitudes large enough to hit the target ribbon, it will occasionally hit the target. The frequency of these collisions can be easily estimated by computing the beam phase space volume occupied by the target ribbon.

In the case that no coupling between the two transverse directions exists, it is sufficient to analyze the transverse position of beam particles in the target direction. Let $x(n)$ be the position of a halo particle after $n$ revolutions at the target location. This can be expressed by

$$
x(n)=W \cdot \sin \Phi(n),
$$

where $W$ is the betatron amplitude (single particle emittance) and $\Phi(n)$ is the betatron phase. The latter can be expressed by $\Phi(n)=\Phi_{0}+n \cdot 2 \pi Q$, where $Q$ is the tune of the machine for the transverse plane under consideration. Since $Q$ is not a simple rational number, the betatron phase randomizes after a few turns, on time scales very small compared to the typical lifetime of a halo particle. If the inner edge of the target ribbon is positioned at a distance $D \leq W$ from the beam core and the transverse dimension of the target is $\delta_{T}$, the probability of a target hit is given by

$$
\begin{aligned}
P_{\mathrm{HIT}}(D, W) & =\frac{1}{2 \pi} \int_{D}^{D+\delta_{T}} \mathrm{~d} x \int_{0}^{2 \pi} \mathrm{d} \Phi \delta(x-W \sin \Phi) \\
& =\left\{\begin{array}{ll}
\frac{1}{\pi}\left(\frac{\pi}{2}-\arcsin \frac{D}{W}\right) & \text { for } D \leq W<D+\delta_{T} \\
\frac{1}{\pi}\left(\arcsin \frac{D+\phi_{T}}{W}-\arcsin \frac{D}{W}\right) & \text { for } W \geq D+\delta_{T}
\end{array} .\right.
\end{aligned}
$$

This result changes if coupling exists between the transverse planes ${ }^{3}$. In this case, the

\footnotetext{
${ }^{3}$ The assumption of strong coupling is realistic since HERA is operated close to the coupling resonance $Q_{x}=Q_{y}$, and since the halo is strongly affected by non-linear effects, like beam-beam interaction, which in general introduce additional cross-plane coupling.
} 


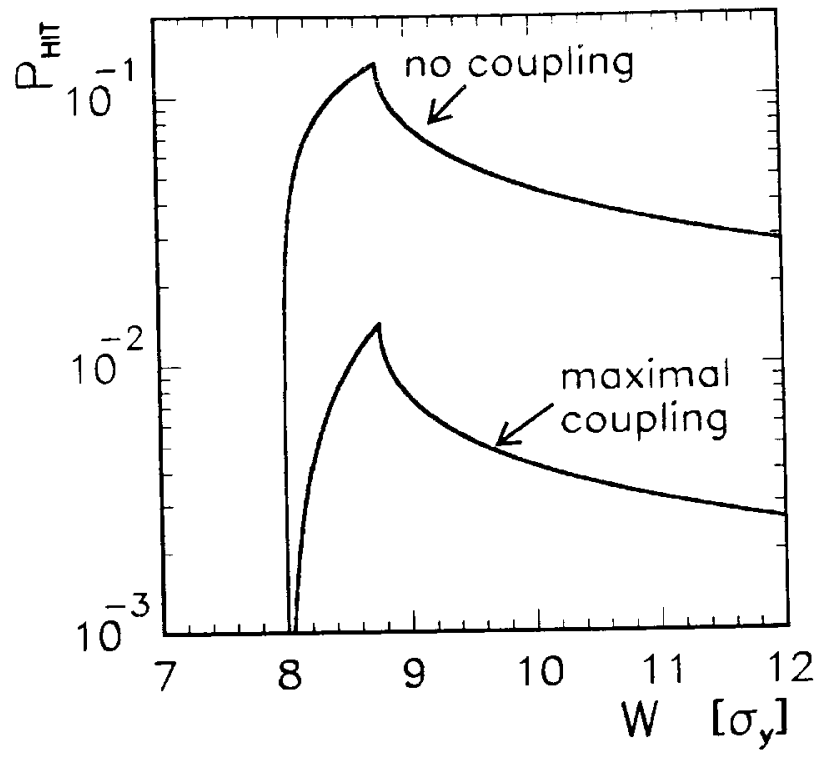

Figure 5: Hit probability per turn for the horizontal target ribbon, positioned at $8 \sigma$ from the beam, as a function of the betatron amplitude for an uncoupled and a fully coupled beam.

amplitude $W$ is not a constant of motion, but beats between two extreme values. The transverse position $x(n)$ depends correspondingly on two phases:

$$
x(n)=W \cdot \sin \Phi(n) \cdot \sin \Psi(n) .
$$

If the coupling strength is large enough, the second phase $\Psi(n)$ also randomizes during the lifetime of halo particles, and the hit probability changes to

$$
P_{\mathrm{HIT}}^{\text {cping }}(D, W)=\frac{1}{\pi} \int_{\arcsin \frac{D}{W^{\prime}}}^{\pi / 2} P_{\mathrm{HIT}}(D, W \sin \Psi) \mathrm{d} \Psi
$$

For illustration, the hit probability is displayed in Fig. 5 for the cases of no coupling and maximal coupling as a function of $W$ for $D=8 \sigma=2.8 \mathrm{~mm}, \delta_{T}=250 \mu \mathrm{m}$. Without coupling, the hit probability is typically of the order of $10 \%$ or less and the wire is hit once in $10 \ldots 100$ turns. Coupling reduces the hit probability by more than one order of magnitude. On the other hand, it increases the number of particles which achieve sufficient betatron amplitudes to hit the target, since large vertical amplitudes gradually transfer into large horizontal amplitudes and vice versa. Coupling is therefore not necessarily expected to harm the target performance.

\section{Interactions:}

A particle which hits the target ribbon may undergo a hard interaction with a target nucleus. The aim of the target optimization is to maximize the achievable rate of such interactions. Since the target material in the test-experiment amounts to $f_{I} \approx 0.4 \%$ of an interaction length (see table 2), interactions take place on the average after 250 target hits. For the hit probabilities discussed above, this implies that - depending on 
the coupling strength - a halo particle is absorbed after several thousand to several ten-thousand turns, corresponding to time scales of roughly $0.1 \ldots 1 \mathrm{~s}$.

The interaction in the target is competing with the absorption of particles on aperture limitations. The target can only absorb particles efficiently if the increase of betatron amplitudes due to diffusion during the mean time before interaction in the wire is smaller than the distance of the inner target edge and the shadow of the aperture limit.

\section{Multiple scattering:}

Before a halo particle is absorbed, it has to traverse the target ribbon on the average $1 / f_{I}=250$ times. Since the target material corresponds to $f_{X}=1.8 \%(\mathrm{Al})$ or $f_{X}=$ $4.2 \%(\mathrm{Cu})$ of a radiation length, the angular width of the halo particles is blown up by the angular smearing due to multiple scattering

$$
\vartheta^{2}=\left(\frac{14 \mathrm{MeV}}{p}\right)^{2} \cdot \frac{f_{I}}{f_{X}}= \begin{cases}(36 \mu \mathrm{rad})^{2} & \text { for } \mathrm{Al} \\ (55 \mu \mathrm{rad})^{2} & \text { for } \mathrm{Cu}\end{cases}
$$

This has to be compared to the natural beam divergence of $\sqrt{\epsilon_{x} / \beta_{x}} \approx 8 \mu \mathrm{rad}$ and $\sqrt{\epsilon_{y} / \beta_{y}} \approx 12 \mu \mathrm{rad}$. Multiple scattering therefore amounts to a smearing of the betatron amplitude by $3 \ldots 5 \sigma(\mathrm{Al})$ and $5 \ldots 7 \sigma(\mathrm{Cu})$. This smearing has to be added in quadrature to the betatron amplitude (typically 8 to $12 \sigma$ ) of the halo particles. The corresponding widening of the beam halo is thus not very severe for the large betatron amplitudes under consideration. Nevertheless, multiple scattering will increase the outward drift of halo particles and decrease the rate of target interactions to some extent, especially for the $\mathrm{Cu}$ target. These losses become much more drastic if tighter collimator settings are considered. One obvious way to reduce the effects of multiple scattering is to increase the angular beam size at the wire position by decreasing the 3 -function at this point.

\section{Energy loss:}

When particles traverse the target material, they do not only undergo multiple scattering but also lose some energy. Since energy loss is continuously compensated in the HERA RF-cavities, the mean energy of the halo particles does not decrease but the $\mathrm{d} E / \mathrm{d} x$ in the target leads to energy (synchrotron) oscillations. These oscillations in turn lead to betatron motions at the target because the dispersion function does not vanish at this position (see table 1).

A halo particle loses typically $0.7 \ldots 0.8 \mathrm{MeV}$ per target hit, i.e. less than a fraction $10^{-6}$ of its energy. For a spatial dispersion of $d_{x}=0.82 \mathrm{~m}$ this amounts to a betatron oscillation amplitude of less than $1 \mu \mathrm{m}$. The effects of energy loss are therefore insignificant, even if hundreds of such synchrotron oscillations were superimposed coherently.

After a target is moved towards the beam, the interaction with the halo leads to a readjustment of the beam profile. The halo which is outside the target position is to a large extent scraped away by the target and the diffusion causes the whole beam profile to readjust 
to the new boundary conditions. We therefore developed two kinds of target models, one in which the steady state situation can be analyzed in detail and a diffusion model to interpret transients and time scales observed after target movements. These models will be briefty described in the following.

\subsection{Single Particle Tracking Model}

This model is useful to interpret interaction rates in the steady state mode and to study their dependence on machine parameters. Single halo particles are generated with betatron amplitudes large enough to allow them to reach the wire target. The particles are then tracked through the proton beam guiding system until they are absorbed on the target or on aperture limitations.

The following processes are included in the model:

- Linear optics: The positions and angles of the halo particles are transported around the ring using single turn transport matrices as derived from the twiss parameters $\alpha$ and $\beta$ of the HERA optics (see table 1). Since the machine is run close to the coupling resonance $Q_{x}=Q_{y}$, we in addition artificially introduce (linear) coupling by assuming a skew quadrupole in a beam waist close to the wire target with focal length $f$ given by

$$
\frac{1}{f}=\frac{2 \pi \cdot \delta}{\sqrt{\beta_{x} \beta_{y}}} .
$$

This leads to betatron amplitudes of halo particles beating between the horizontal and vertical direction with frequency $\delta / T$, with $T=21 \mu$ s being the revolution time of a proton. Due to non-linear effects dominating the halo dynamics, the effective value for $\delta$ can be significantly larger than the difference between horizontal and vertical tunes (which is of the order $10^{-3}$ ).

- Diffusion: The diffusion process is taken into account by introducing an outward drift velocity ${ }^{\prime} D(W)$ of halo particles at betatron amplitudes $W$. For every single turn, the betatron amplitudes are randomly increased by a value between 0 and $2 v_{D}(W) \cdot T$. Since it is known that the diffusion speed is very steeply increasing with amplitude, the drift velocity is parameterized as

$$
v_{D}(W)=v_{D}\left(W_{0}\right) \cdot\left(\frac{W}{W_{0}}\right)^{\kappa},
$$

where $W_{0}$ corresponds to a typical position of the target ribbon, for our tests $W_{0}=$ $10 \sigma$. The parameters $W_{0}$ and $\kappa$ are not known a priori for the extreme halo. Some measurements exist, however, for much shorter distances around $5 \sigma[12]$.

- Target hits: If a proton hits a target, a multiple scattering is performed, using a purely Gaussian distribution for the multiple scattering angle distribution. At the same time the energy of the particle is reduced by the mean $\mathrm{d} E / \mathrm{d} x$ value and a synchrotron oscillation is launched. For every target hit, the particle is absorbed by an interaction with a nucleus with a probability according to the fractional interaction length of the target material $(0.4 \%)$. 
- Losses: If a particle hits a collimator jaw it is immediately absorbed ${ }^{4}$. We neglect the fact that particles may be scattered back from the jaws into the acceptance of the beam pipe: these particles are lost immediately on secondary collimators or are absorbed on other aperture limitations within a few turns.

Since the model uses a number of machine parameters which are not known $\left(v\left(W_{0}\right), \kappa\right)$ or poorly known (shape of the aperture, coupling strength) its predictive power is small. The main purpose is to interpret the measurements of interaction rates in terms of the unknown parameters and therefore gain information on the dynamics of the beam halo. Once a reasonable range of parameter values is identified, the model can serve to optimize the design of the internal target and of the machine optics at the target location.

\subsection{Diffusion Model}

In reference [12] a simple analytical diffusion model is developed which allows to compute the time dependence of the beam profile after movements of the collimators. The diffusion equation reads

$$
\frac{\partial}{\partial t} f(Z, t)=R \frac{\partial}{\partial Z}\left(Z \frac{\partial}{\partial Z} f(Z, t)\right)
$$

where $f(Z, t)$ is the beam profile and $Z=W / W_{C}$ is the scaled betatron amplitude, normalized to the maximal betatron amplitude given by the collimator position $\left(W_{C}\right)$.

The diffusion constant $R$ is assumed to be independent of $W$, an assumption which is in general not fulfilled, since diffusion effects are strongly dependent on the betatron amplitude [11]. As shown in [12], it is nevertheless possible to describe the dynamics of the halo close to the collimators by the simplified equation (8), if one interprets $R$ as the local diffusion constant close to the collimators.

The diffusion equation can be solved using the boundary conditions of vanishing particle current at $W=0$,

$$
\left.Z \cdot \frac{\partial}{\partial Z} f(Z, t)\right|_{Z=0}=0
$$

and vanishing particle density at the collimator jaw,

$$
f(Z=1, t)=0
$$

by expansion into first type Bessel functions:

$$
f(Z, t)=\sum_{n} c_{n} J_{0}\left(\lambda_{n} \sqrt{Z}\right) \exp \left(-\lambda_{n}^{2} \frac{R}{4} t\right) .
$$

The constants $\lambda_{n}$ denote the $n$-th zeros of $J_{0}$. For any initial beam profile $f_{0}(Z)$, the parameters $c_{n}$ can be computed using the orthogonality of the Bessel functions:

$$
r_{n}=\frac{1}{J_{1}^{2}\left(\lambda_{n}\right)} \int_{0}^{1} f_{0}(Z) J_{0}\left(\lambda_{n} \sqrt{Z}\right) \mathrm{d} Z
$$

\footnotetext{
${ }^{4}$ We assume that all other potential aperture limitations in the beam guiding system are totally shadowed by the collimators.
} 
Analytical expressions are given in reference [12]. Since $\lambda_{n}$ is increasing almost linearly with $n$, the high $n$ components are strongly damped away by the time dependence in equation (12). Asymptotically the beam profile has the shape $J_{0}\left(\lambda_{0} \sqrt{Z}\right)$ and the asymptotic beam lifetime is $\tau=4 /\left(\lambda_{0}^{2} R\right)$. The $n=1$ and $n=2$ terms have lifetimes which are already 5.3 and 13 times smaller.

The transient behaviour after movements of the collimator can be computed analytically in the following way:

1. The beam profile just before the collimator movement is denoted by $f_{0}(Z)$. Typically the beam has reached an equilibrium state, so that $\left.f_{0}(Z)=J_{0}\left(\lambda_{0} \sqrt{(} Z\right)\right)$.

2. The collimator is moved from $W_{C}$ to $\tilde{H}_{C}$. This implies a change in the scale $Z$ :

$$
Z \rightarrow Z \cdot \frac{W_{C}}{\dot{W}_{C}}
$$

and in these new coordinates the initial beam profile immediately after the collimator motion reads

$$
\tilde{f}_{0}(Z)= \begin{cases}f_{0}\left(Z \cdot \tilde{W}_{C} / W_{C}\right) & \text { for } Z \cdot \tilde{W}_{C} \leq W_{C} \\ 0 & \text { otherwise }\end{cases}
$$

3. This function is analytically re-expanded into Bessel functions according to equation (12), and the time dependence of the new beam profile in turn follows from equation (11).

4. Further collimator movements make use of the initial profile as computed in step 3 and are treated in analogy as in steps 2,3 .

Strictly speaking, this model only applies for the case of boundaries defined by collimators. If the collimator is replaced by the target, the beam profile can extend beyond the target edge, since the target material is semi-transparent. The boundary condition in equation $(10)$ is therefore smeared out. As will become clear in section 6 , the observed diffusion rates lead, however, to lifetimes orders of magnitude larger than the $\mathcal{O}(1 \mathrm{~s})$ absorption time of halo particles by the target. For components of the halo which evolve according to the above diffusion processes, the target can therefore in very good approximation be replaced by a collimator jaw.

\section{Interaction Rates in the Target}

The results presented in this and the following sections were obtained from data taken during the 1993 luminosity operation of HERA. The target tests resulted in a total of 55 hours of data taking in 9 different fills of the proton ring. During the majority of the run time (52 hours) the machine was operated in stable ep luminosity mode. The rest of the tests were performed without electrons stored in the machine or during the injection phase of the electron ring. The proton current was typically more than one order of magnitude below the design current. The rate capability of the internal target can therefore be expected to improve by at least one order of magnitude during the next years. 
In order to provide a measure of target performance which is independent of the current in the machine, we will define the target efficiency $\epsilon_{T}$ as the ratio of the rate of interactions in the target to the total rate. at which protons are lost from the beam. At the $160 \mathrm{~mA}$ design current and a (conservative) current lifetime of $100 \mathrm{~h}, 6 \cdot 10^{7}$ protons are lost per second; the $30 \mathrm{MHz}$ rate of the $B$ experiment proposed in [6] requires a minimum target efficiency of $50 \%$

\subsection{Wire Scans}

The primary goal of the internal target test was to demonstrate that high target efficiencies well above $50 \%$ can be achieved. Fig. 6 shows a typical target scan where the horizontal aluminum ribbon (see Fig. 3) was slowly moved towards the beam. The upper plot displays the distance of the target ribbon to the beam (in units of r.m.s. beam widths) as a function of time. The lower diagram shows the history of the trigger rate. With the wires in their rest position outside the aperture, a background counting rate of about $4 \mathrm{kHz}$ is observed, mainly due to beam-wall interactions. It can clearly be seen that at about $12 \sigma$ the target leaves the collimator shadow and the trigger rate suddenly starts to rise. At a distance of $8 \sigma$ the trigger rate reaches $200-300 \mathrm{kHz}$. It remained at that level for about one hour. During this time the rate changed only once due to a beam adjustment. Finally the wire was retracted in steps and the rate decreases.

Besides the overall increase of trigger rate with decreasing distance of target and beam, the rates exhibit extra structure connected to target movements. This can be seen in a blowup of a small time interval of the scan in Fig. 6, as presented in Fig. 7. Directly after a target movement towards the beam, the trigger rate rises sharply and then decays within a few minutes to a new steady state value. This behaviour is qualitatively explainable by the fact that the target in its new position scrapes away the outer halo until the beam phase space population has reached a new equilibrium. The inverse behaviour can be seen if the target is retracted (see Fig. 6). In this case the rates suddenly drop and then rise again until the halo is refilled. These aspects will be discussed in more detail in section 6 .

\subsection{Evidence for Interactions in the Target}

The observation of large trigger rate is, strictly speaking, not a complete proof for the proper operation of the halo target. It could be imagined, e.g., that the target beam interaction somehow produces an enormous enhancement of the beam halo and an increased background all around the storage ring; the trigger counters could then be fired by particles generated in upstream beam wall interactions. There are however a number of convincing proofs for the wire as the dominant source of interactions:

- There is no indication of any excessive target correlated background in the other HERA experiments (compare section 10).

- The fraction of triggers accompanied by VETO signals drops drastically when the wire is moved in. This is demonstrated in Fig. 8a where the trigger rate (for a different run, see also Fig. 11) is shown together with the rate of hits of any VETO counter as well as of the coincidence of all four VETO counters. When the target is retracted, the 


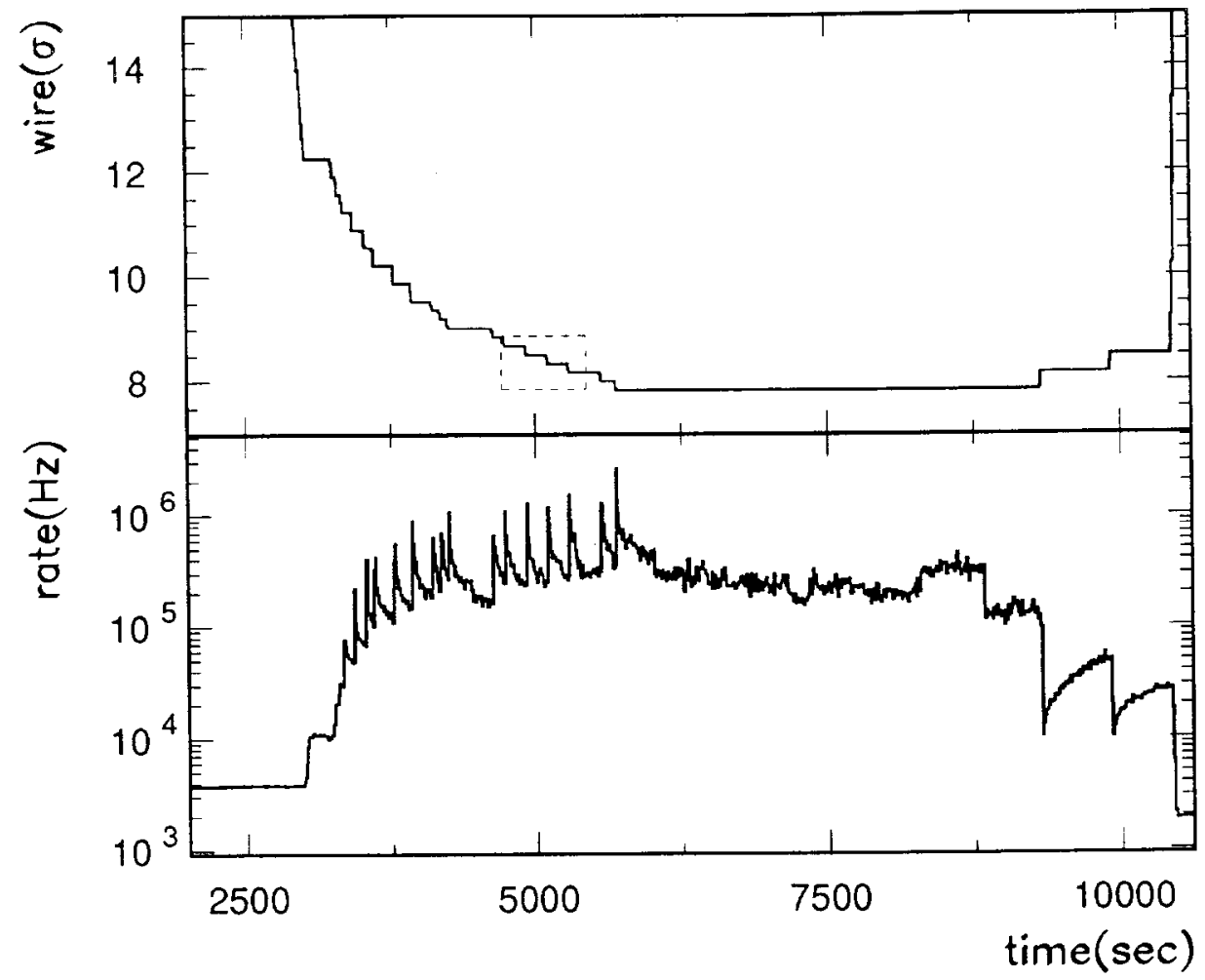

Figure 6: Target (horizontal Al ribbon) distance to beam center measured in r.m.s. beam widths and trigger rate as a function of time. The region inside the dashed box is blown up in Fig. 7.

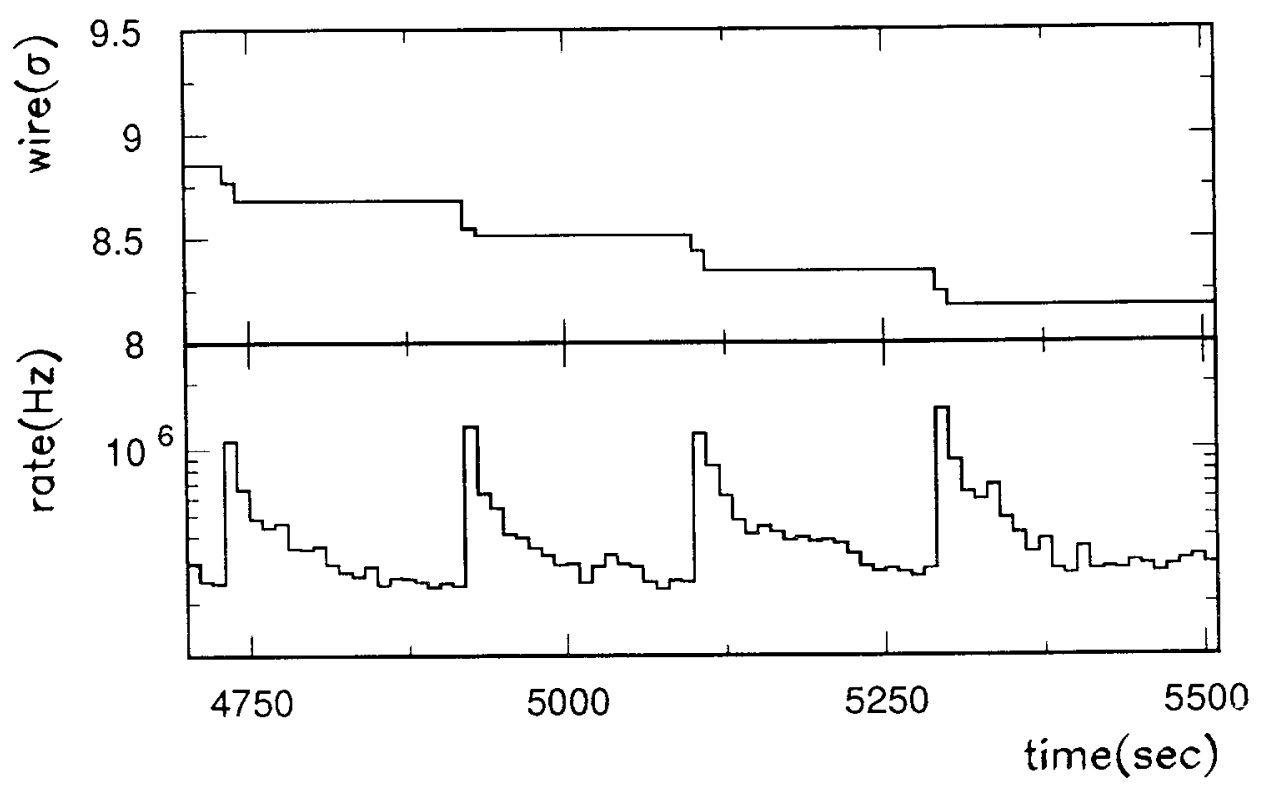

Figure 7: Blow-up of a short section of the scan in Fig. 6. One bin corresponds to $10 \mathrm{~s}$. 

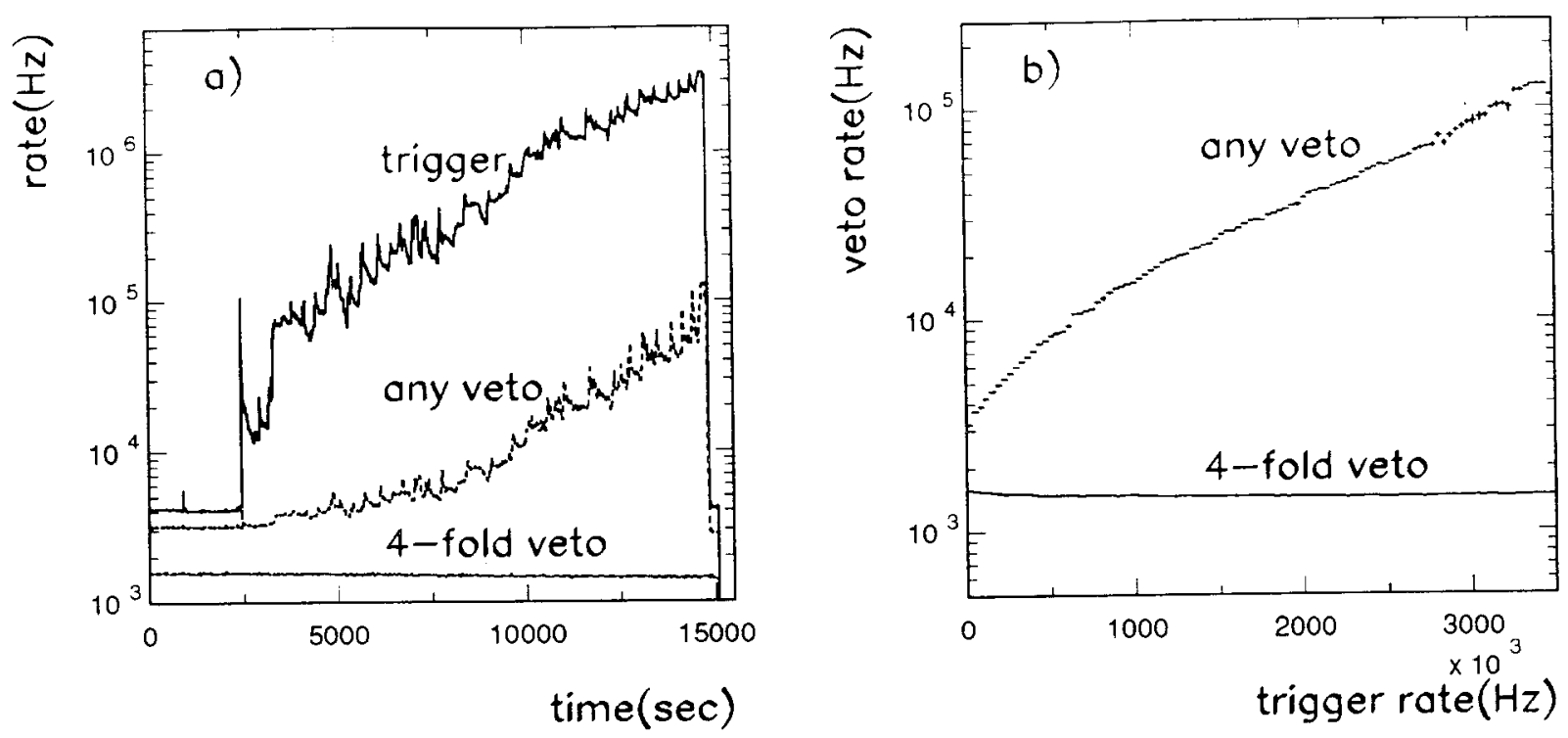

Figure 8: a) Trigger rate and rate of hits of any VETO counter, and coincidence rate of all four VETO counters as a function of time. b) The same veto rates but as function of the trigger rate. The data stem from the scan in Fig. 11.

majority of all triggers are accompanied by VETO signals. In $40 \%$ of the events even all four VETO counters are set in coincidence. We attribute these background events to upstream interactions. When the target touches the beam halo, the fraction of events with a VETO immediately drops to the percent level. Triggers are therefore not due to events originating upstream of the target. It is interesting to note that the rate of single VETO hits is highly correlated with the trigger rate but stays suppressed by roughly 2 orders of magnitude. This is shown in Fig. $8 \mathrm{~b}$ where the veto rates are plotted as a function of the trigger rate. ${ }^{5}$ This indicates that occasionally single particles are backscattered when interactions take place in the target and hit single VETO counters. The single VETO events are therefore to a large extent due to interactions in the target and not to background events. The coincidence rate of all VETO counters, however, is uncorrelated with the trigger rate and its relative magnitude drops to a negligible level when the target is moved into the halo (Fig. 8b). Interactions in the target thus do not produce large particle densities in the backward hemisphere (as expected), and these fourfold VETOs are entirely due to background events, typically high multiplicity showers originating a few meters upstream of the target and hitting all counters. The fact that the fourfold VETO rate is stable or even slightly decreasing implies that the internal target does not create any additional background for the test experiment.

- The most striking evidence in favor of real target interactions is the observation of tracks in the drift chambers which point towards the target. Fig. 9 shows an example of an event with reconstructed tracks in the chambers. Fig. 10 displays distributions of the horizontal and vertical position of reconstructed tracks at the longitudinal

\footnotetext{
${ }^{5}$ The single VETO rate does not increase linearly with the trigger rate. This is due to multiple interactions in single bunch crossings, as will be discussed in section 5.3
} 


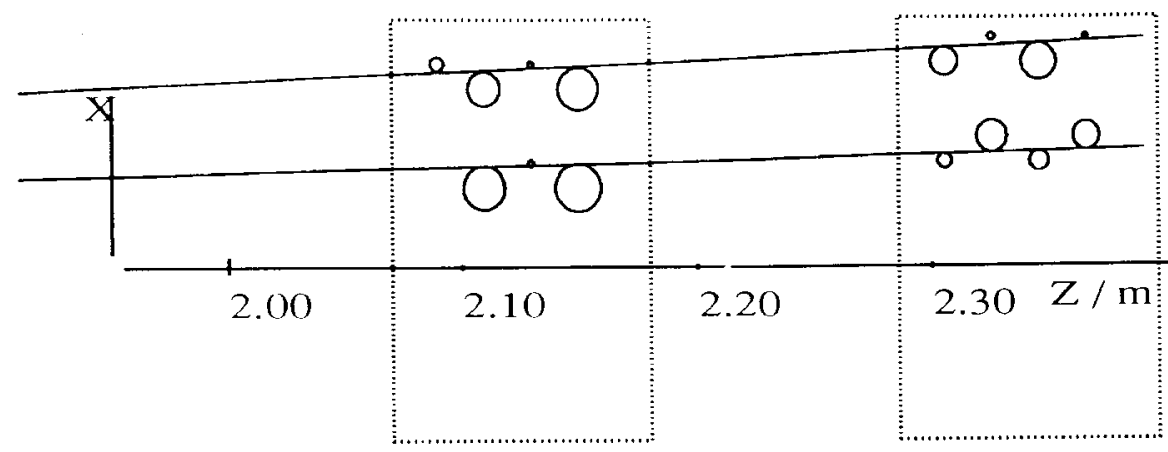

Figure 9: Example of a target interaction with tracks in the acceptance of the drift chambers. The wire hits are indicated by circles of constant drift time. The straight lines represent the reconstructed tracks.

location of the target for the cases that the upper (lower) horizontal ribbon is positioned inside the halo and for retracted target (background). The track intercepts are centered around the position of the target ribbons. The widths of the distributions $(\approx 7 \ldots 9 \mathrm{~mm})$ are consistent with the expected resolutions which are strongly affected by multiple scattering in the beam pipe exit window (see Fig. 2). The vertical offset (of $4.4 \pm 0.5 \mathrm{~mm}$ ) between interactions in the upper and lower target can be clearly seen and agrees with the vertical offset between the two target positions. As expected, in horizontal direction no such offset is visible. The mean horizontal and vertical impact parameters agree well with the measured beam position. Background tracks show much broader distributions of impact parameters which can be expected because their origins should be spread over many meters upstream of the target. The resolution of the drift chambers is not high enough to assign tracks to targets on an event by event basis. The tracking system will therefore be upgraded in the future.

\subsection{Determination of Peak Interaction Rates}

The run in Fig. 6 was taken with a very long proton beam lifetime of $\approx 400 \mathrm{~h}$, with a corresponding steady state proton loss rate of $1.2 \mathrm{MHz}$. Much higher rates are achievable with lifetimes of $100 \mathrm{~h}$ or below. In Fig. 11 the target scan with the highest trigger rates achieved up to now is displayed. In this run, the lifetime of the proton beam was rather low (around $20 \mathrm{~h}$ ). The trigger rate reaches values of more than $3 \mathrm{MHz}$.

The actual rate of proton interactions in the target ribbon is significantly higher than the trigger rate, once $\mathrm{MHz}$ rates are measured. The reason is that the trigger cannot resolve multiple interactions in a single bunch crossing. The peak trigger rate is therefore $4 \mathrm{MHz}$ (corresponding to the bunch crossing rate for 84 bunches with $47.3 \mathrm{kHz}$ revolution frequency). Assuming that the number of interactions per BX obeys Poisson statistics, the trigger rate $R$ can be related to the bunch crossing rate $R_{0}$, the mean number of interactions per $\mathrm{BX}$, $n_{\text {Int }}$, and the acceptance of the trigger counters, $\epsilon$ :

$$
R=R_{0}\left(1-\epsilon^{-\epsilon n_{I n t}}\right)=\left\{\begin{array}{ll}
R_{0} \epsilon n_{I n t} & \text { for } \epsilon n_{I n t} \ll 1 \\
R_{0} & \text { for } \epsilon n_{I n t} \gg 1
\end{array},\right.
$$




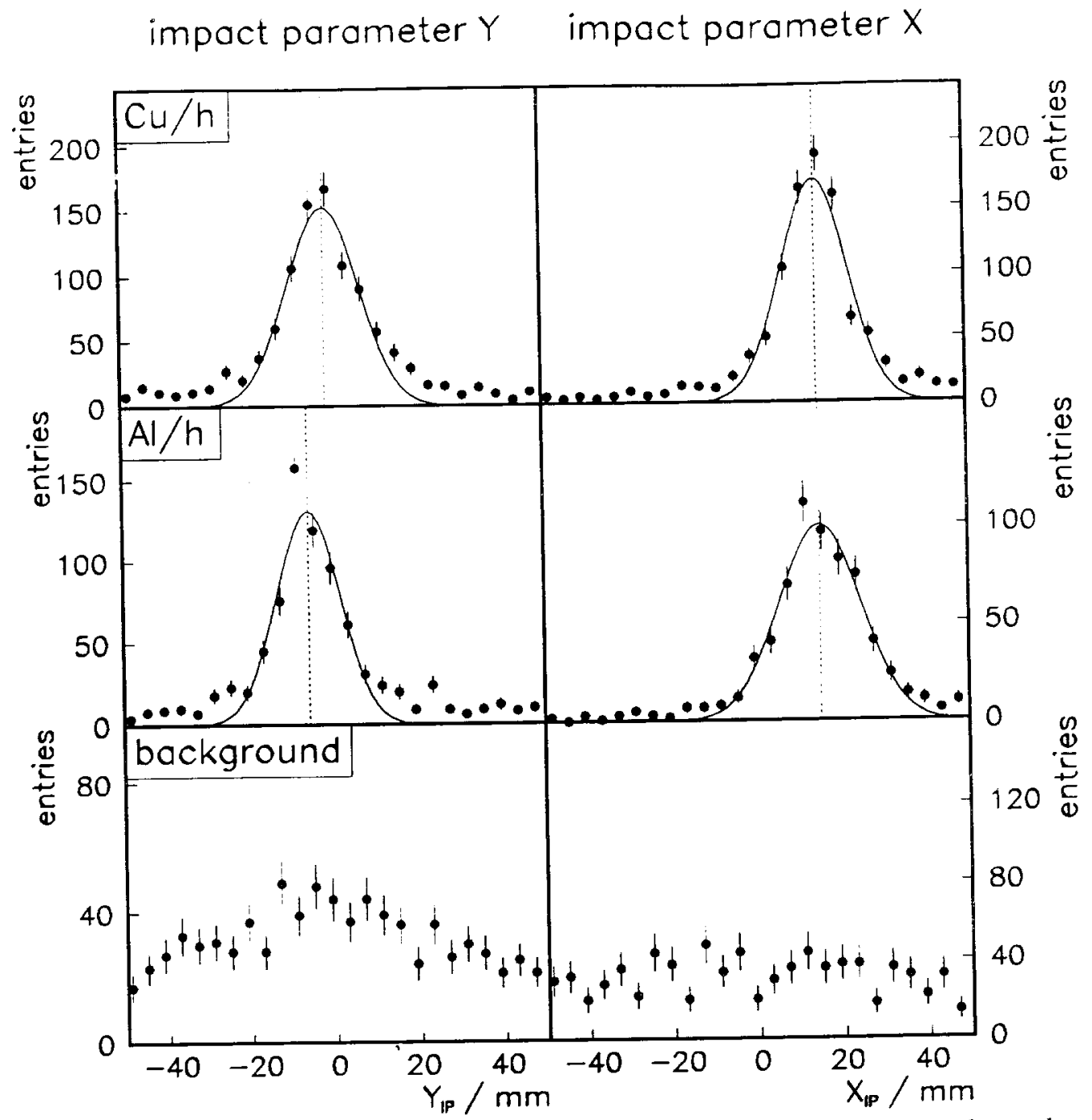

Figure 10: Vertical (left) and horizontal (right) position of reconstructed tracks at the longitudinal position of the target for interactions in the upper (lower) horizontal target ribbon and for retracted targets (background). The curves are Gaussian fits to the central parts of the distributions.

or

$$
n_{\text {Int }}=-\frac{1}{\epsilon} \ln \left(1-\frac{R}{R_{0}}\right) .
$$

The latter quantity is displayed in Fig. 11 for the high rate scan. It can be seen that up to two interactions per BX were produced at the highest trigger rates. The corresponding interaction rates are roughly $8 \mathrm{MHz}$.

The above analysis depends on the assumption that Poisson statistics can be applied for the number of interactions per BX. As will become clear in section 8, this assumption is in general not exactly correct. mainly due to fluctuations of the rates produced by the individual bunches. An independent measurement of $n_{\text {Int }}$ serving as a cross check can be obtained by studying the rate of a counter with much smaller efficiency $\epsilon$ than the trigger counters, such that even for $n_{\text {Int }}=2 \ldots 3$ the quantity $\epsilon n_{\text {Int }}$ is still well below one. Then, 


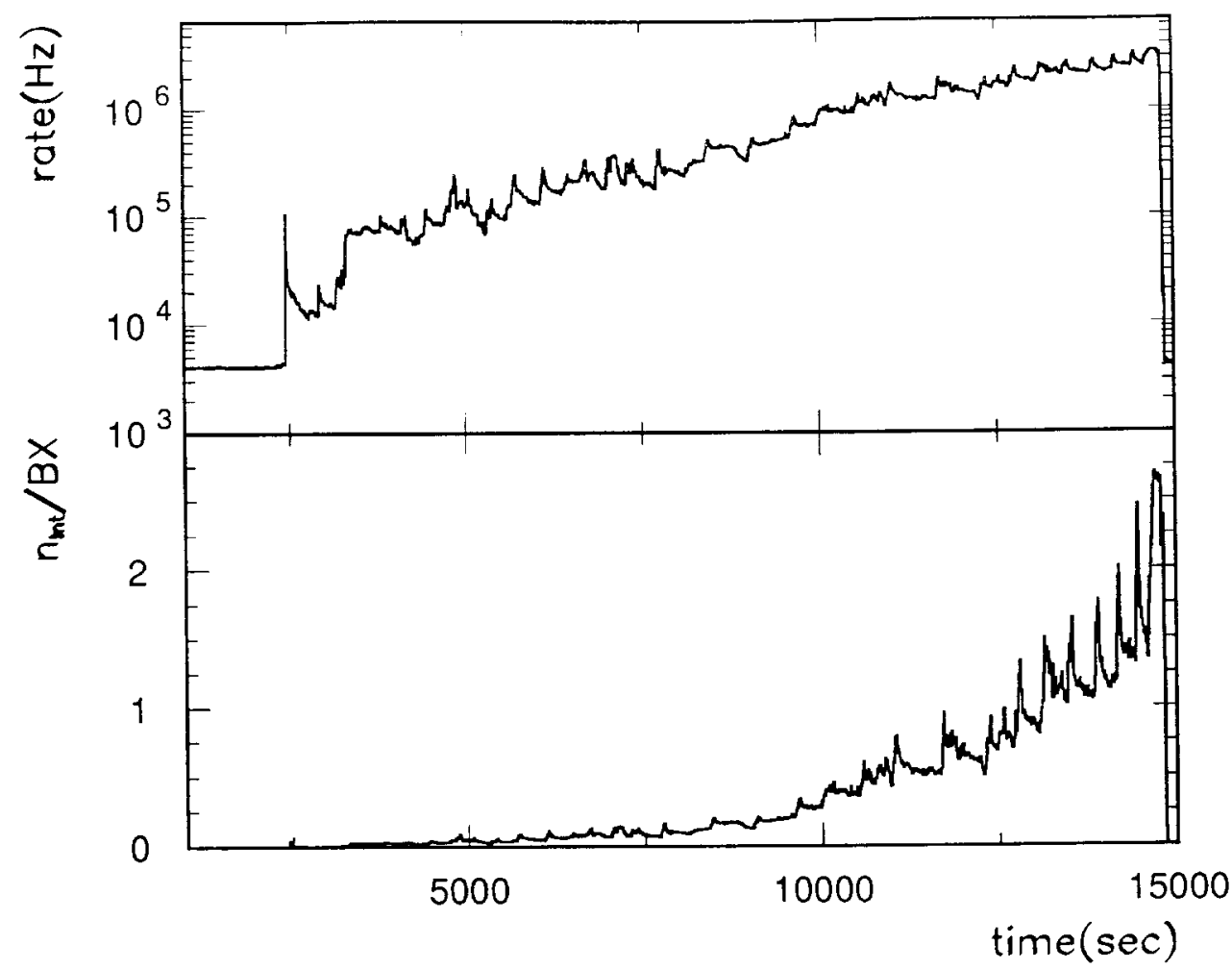

Figure 11: High rate target scan using the vertical $C$ u target together with the mean number of interactions per $B X$.

the rate of this counter is always proportional to the target interaction rate (eq. (15)). The scintillator hodoscopes are not suitable for this purpose, since their event acceptance is still too large. The VETO counters, however, serve this purpose well, since they are hit rarely (in roughly $1 \%$ of all interactions) by backscattered particles produced in the target (see section 7). The trigger probability $\epsilon_{V}$ in these counters is not known a priori, and is hard to determine reliably from Monte Carlo simulations. However, $\epsilon_{V}$ can be measured by comparing the VETO rate to the trigger rate in a regime where the trigger rate is still a good estimate for the interaction rate. In Fig. 12a the ratio of the rate of VETO hits and the trigger rate is shown for the scan in Fig. 11 as a function of the trigger rate. Initially the ratio is big since the target is retracted and VETO hits are exclusively due to background events. As soon as the trigger rate increases due to real interactions in the target, the ratio drops sharply to the level of about $1 \%$, stays constant up to a trigger rate of $1.5 \mathrm{MHz}$ and then starts to rise again. The latter effect is due to saturating trigger rates. If, on the other hand, the trigger rate is translated into interaction rates using the poissonean correction in equation (16), the ratio stays constant up to highest trigger rates (also shown in Fig. 12a, curre 3 ). This indicates that the estimate of $n_{\text {Int }}$ can be used with confidence.

These results are further corroborated by Fig. $12 \mathrm{~b}$ which displays the fractions, $f_{k}$, of triggers with a $k$-fold coincidence $(k=1 \ldots 4)$ of the scintillator hodoscopes as a function of the trigger rate. The fraction $f_{4}$ increases quite strongly with increasing rate, an effect which is naively expected because fourfold coincidences become more probable when the mean number of interactions per BX increases. A more quantitative interpretation is possible 

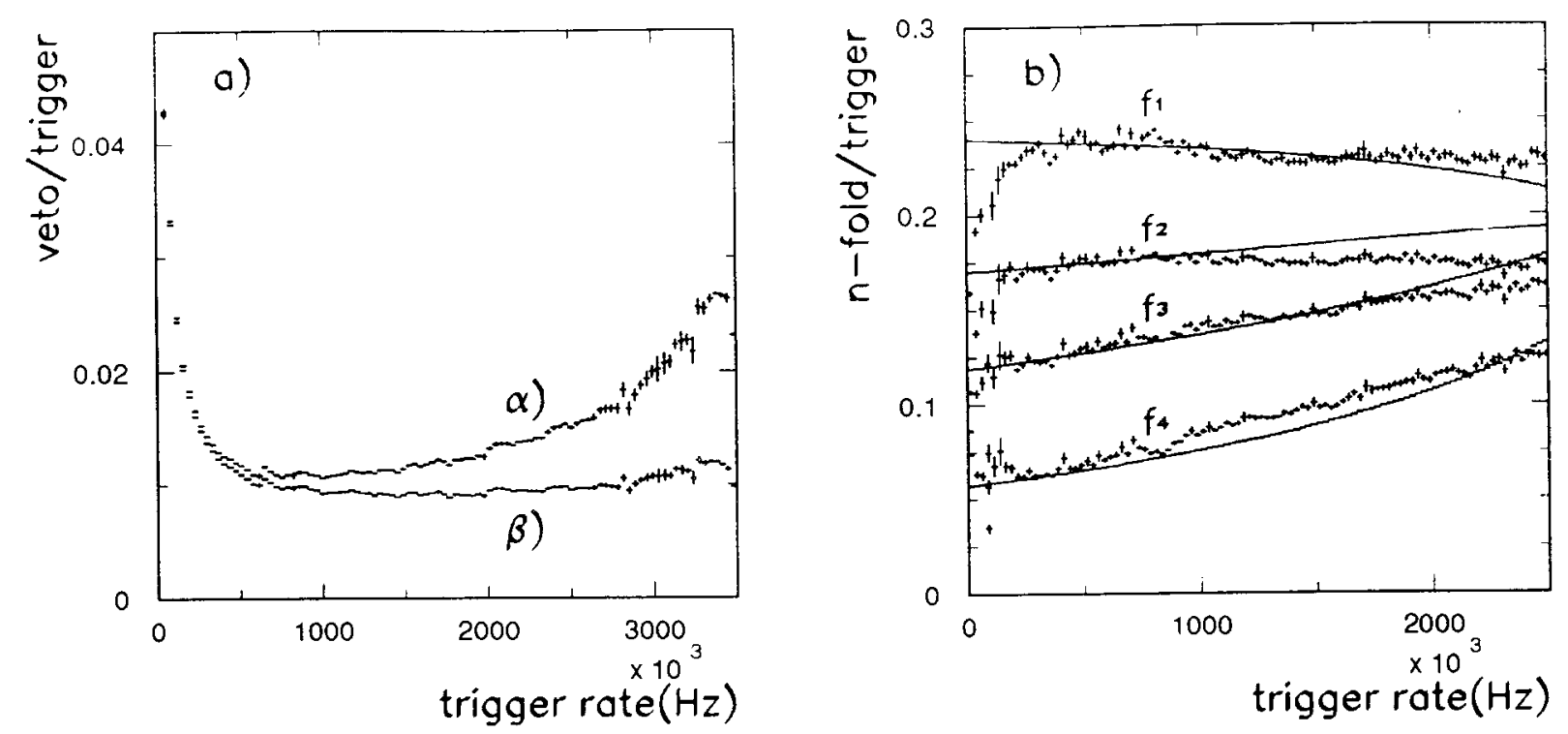

Figure 12: a) Ratio of VETO and trigger rate ( $\alpha$ ) and of VETO and interaction rate ( $\beta$ ) for the scan in Fig. 11. b) Relative rate of $k$-fold coincidences of the telescopes as a function of the trigger rate together with predictions of a statistical model (see text).

under three assumptions (which are all only approximately fulfilled):

1. The number of interactions per BX obeys Poisson statistics.

2. The rate of $k$-fold coincidences is the same for all combinations of $k$ scintillator hodoscopes.

3. The background contributions can be neglected.

If $p_{k}$ are the probabilities for exactly $k$ scintillators hit in coincidence for single interactions (they can be measured at small trigger rates where multiple interactions per BX are extremely rare), the probability, $Q_{k}^{n}$, for a $k$-fold coincidence in $n$ superimposed but uncorrelated interactions can then be computed by the recursion relation

$$
\begin{aligned}
Q_{k}^{0} & =\delta_{k 0} \\
Q_{k}^{n+1} & =\sum_{i=0}^{k} Q_{i}^{n} \cdot \sum_{j=k-i}^{k} p_{j} \cdot \frac{\left(\begin{array}{c}
N-i \\
k-i
\end{array}\right)\left(\begin{array}{c}
i \\
j-k+i
\end{array}\right)}{\left(\begin{array}{c}
N \\
j
\end{array}\right)},
\end{aligned}
$$

where $N(=4)$ is the number of scintillator hodoscopes. The fraction, $f_{k}$, of $k$-fold coincidences follows from

$$
f_{k}=\frac{\mathrm{e}^{-n_{I n t}}}{1-\mathrm{e}^{-n_{I n t}}} \cdot \sum_{i=1}^{\infty} \frac{n_{I n t}^{i}}{i !} Q_{k}^{i} .
$$

The prediction of this simple model is also shown in Fig. 12b using the coincidence probabilities measured at low rates $\left(p_{0}=0.41, p_{1}=0.24, p_{2}=0.17, p_{3}=0.12, p_{4}=0.06\right)$. The agreement with the measured fractions is satisfactory within the simplifications of the model at rates high enough that the background rate $(\approx 4 \mathrm{kHz})$ can be neglected. 

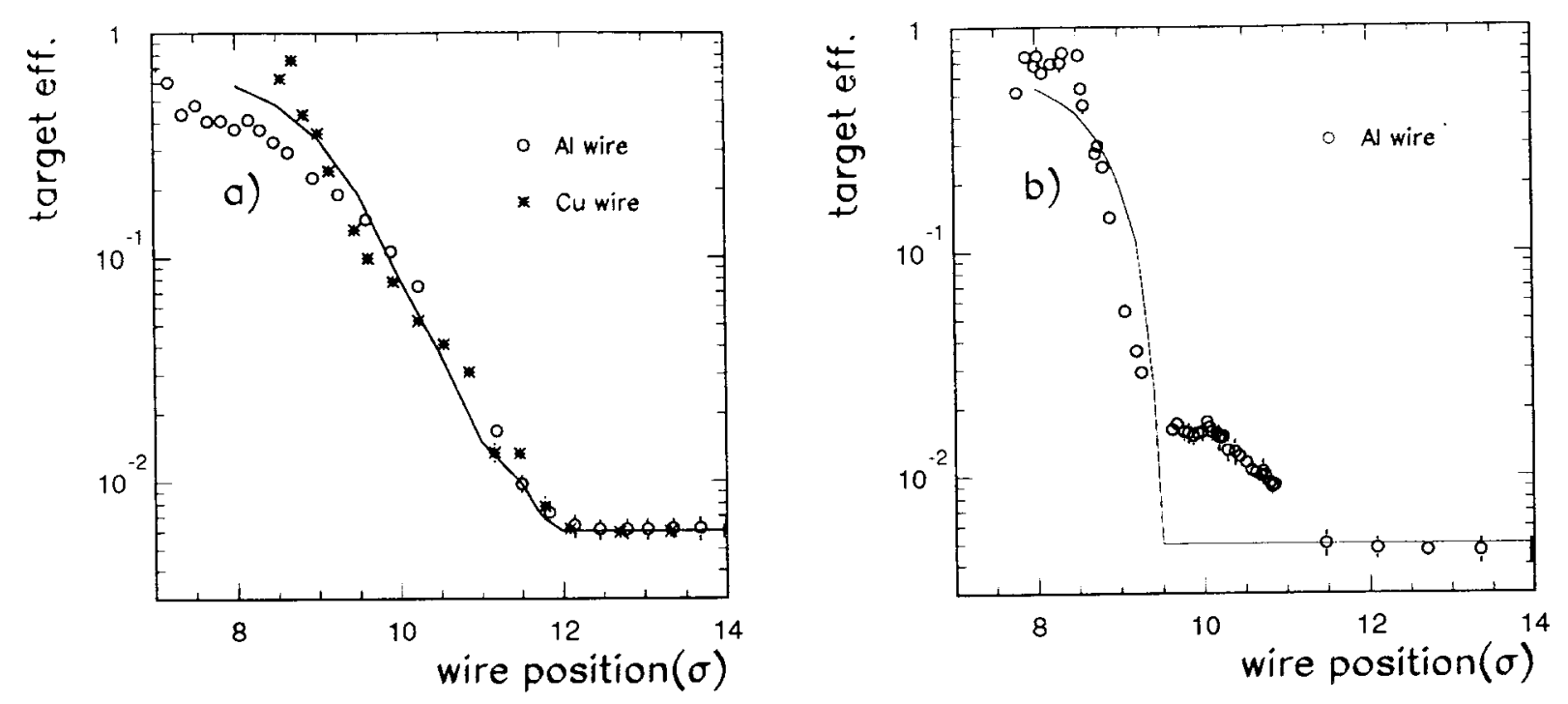

Figure 13: Target efficiency as a function of the distance of target and beam center (measured in r.m.s. beam widths) for scans using (a) the horizontal Al ribbon (circles) and the horizontal $C u$ ribbon (asterices) in the same proton fill, and (b) the horizontal Al ribbon in a different fill.

\subsection{The Target Efficiency}

The total trigger rate discussed in section 5 depends on machine parameters like the total current and the beam lifetime. In order to quantify the quality of the internal target, the target efficiency $\epsilon_{T}$ was introduced. It is defined by the fraction of protons interacting in the target, compared to all protons leaving the machine. The target efficiency can be computed from the beam lifetime, $\tau_{p}$, the total number of stored protons, $N_{p}$, and the interaction rate, $\dot{N}$. which is derived from the trigger rate according to equation (16) (and $\dot{N}=n_{\text {Int }} \cdot R_{0}$ ):

$$
\epsilon_{T}=\frac{\tau_{P}}{N_{p}} \cdot \dot{N}
$$

The target efficiency can only be estimated reliably when the rates have reached an equilibrium state. The spikes of the trigger rates observed after moving a target towards the beam correspond to momentary reductions of the beam lifetime. Given the finite precision of the proton current measurement from beam pick-up signals, this reduction cannot be measured on the short time scales of the spikes. For equilibrium states the lifetime was estimated by exponential fits of the beam current over time intervals which were by an iterative procedure adjusted to correspond to a fixed (small) fraction of the beam lifetime. For target efficiencies of $50 \%$ and the machine running at design currents, the rates necessary for a $B$ experiment require that the beam lifetime does not exceed $100 \mathrm{~h}$.

In Fig. 13a the target efficiency is displayed as a function of the distance of target and beam center (measured in r.m.s. beam widths) for a scan in which first the horizontal Al ribbon (this scan is shown in Fig. 6) and afterwards the horizontal $\mathrm{Cu}$ ribbon was moved towards the beam. The beam lifetime was about $400 \mathrm{~h}$. As soon as the target leaves the 

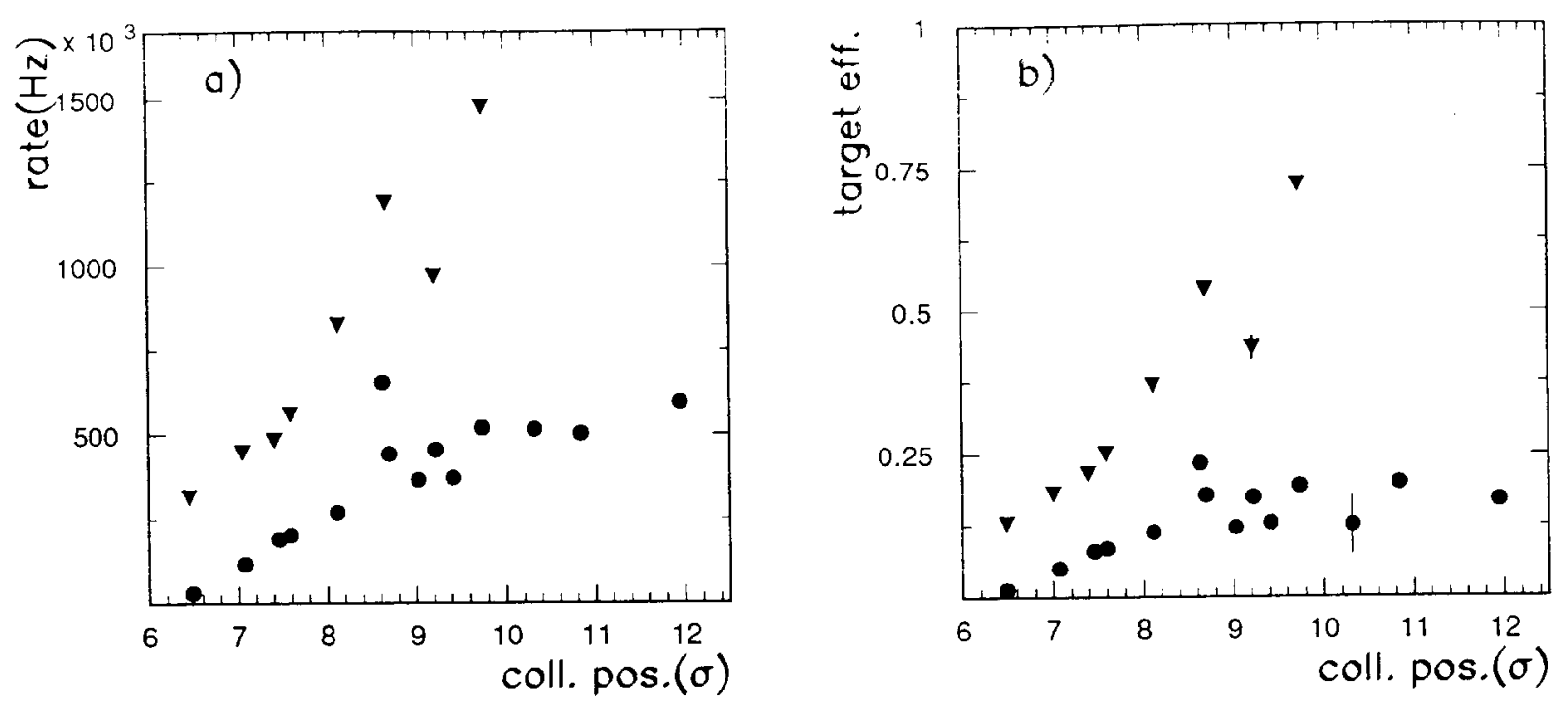

Figure 14: Trigger rate (a) and target efficiency (b) as a function of the horizontal position of the vertical collimator jaw (measured in r.m.s. beam widths) for two fixed positions of the vertical Al ribbon (dots: $\approx 6.5 \sigma$; triangles: $\approx 5 \sigma$ ).

shadow of the collimator jaws (positioned around $12 \sigma$ ) the target efficiency starts to rise quickly. At distances of $7-9 \sigma$ efficiencies $>60 \%$ are reached for both target materials.

The detailed dependence of the target efficiency on the target position is different from fill to fill and depends especially on the exact structure of aperture limitations. Fig. 13b shows another example of a scan of the horizontal Al ribbon. The beam lifetime was $\approx 200 \mathrm{~h}$ in this case. The target leaves the collimator shadow at a distance of $9.5 \sigma$; the efficiency then increases sharply and saturates at $70 \%$ already at a distance of $8.5 \sigma$. In Fig. 14 the importance of the collimator position was studied systematically by driving in the vertical collimator jaw with two fixed positions of the vertical Al target ribbon. It can be seen that the observed rates (and target efficiencies) increase rapidly when the collimator jaw is moved out.

The target efficiency can be interpreted in the framework of the single particle tracking model described in section 4.2. The relatively slow increase of $\epsilon_{T}$, observed in Fig. 13a, can only be explained, if the drift velocity is large and strongly increasing with betatron amplitude (a behaviour which is qualitatively expected [11]). The line in Fig. 13a represents the model predictions ${ }^{6}$ for $v_{D}(10 \sigma)=20 \sigma / \mathrm{s}$ and $\kappa=23$, using the aluminum target and assuming the collimator jaws to be positioned at $12 \sigma$. These parameters can also describe the measurement in Fig. 13b reasonably well if the vertical collimator jaw is moved to $9.5 \sigma$. The slight increase of the target efficiency at distances between 11 and $9.5 \sigma$ may be due to the detailed structure of the aperture boundary. No attempt was made to fit this structure with the model. The difference between the target efficiency of the $\mathrm{Al}$ and $\mathrm{Cu}$ target, visible in Fig. 13a. is probably due to a change of conditions between the two scans. Since the two ribbons have the same width and represent the same fraction of an interaction length, the

\footnotetext{
${ }^{6}$ The background contribution to the target efficiency, taken from the data in case the target ribbon is positioned beyond the collimator shadow, is added to the results of the model.
} 


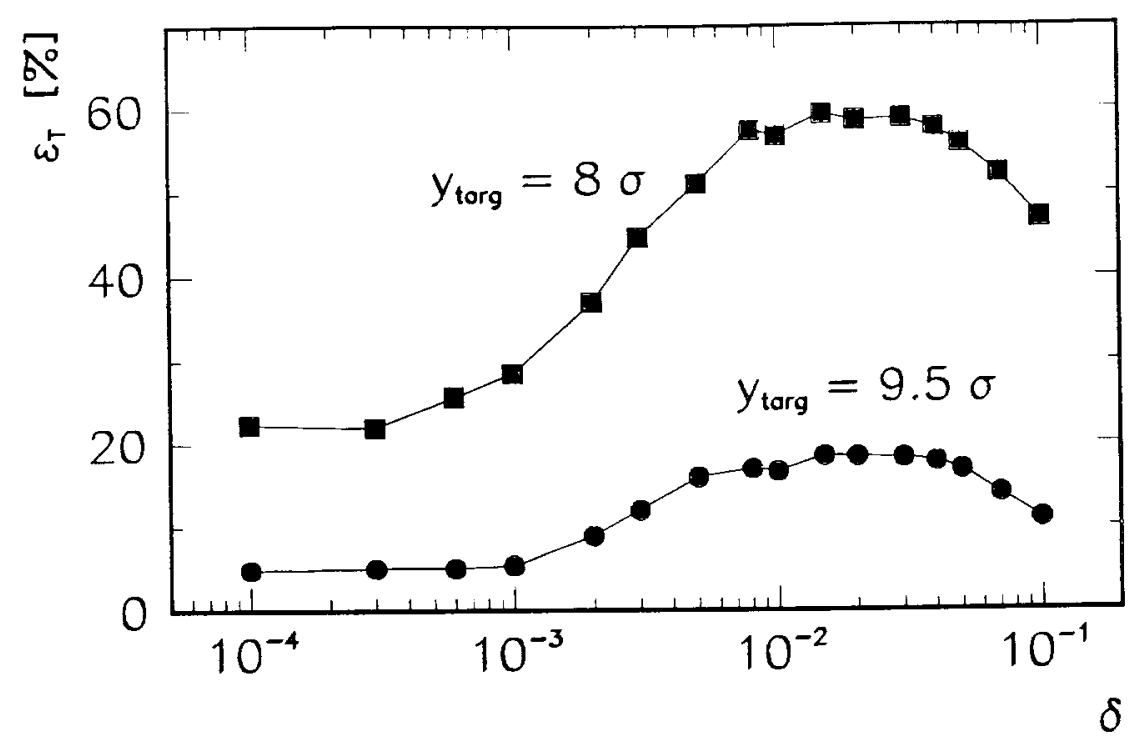

Figure 15: Prediction for the target efficiency for target distances of $8 \sigma(9.5 \sigma)$ as a function of the coupling strength. All other parameters are identical to those used in Fig. $13 a$.

target efficiency of the $\mathrm{Cu}$ ribbon can under identical conditions never be larger than that of the Al target due to the larger multiple scattering in the Cu ribbon.

The target efficiency is not only affected by the structure of the aperture limitation and the magnitude of the drift velocity, but it is also sensitive to the cross plane coupling in the beam. The predictions in Fig. 13 correspond to a large coupling strength of $\delta=0.01$. In Fig. 15 the target efficiency in Fig. 13a, at a fixed target position of $8.5 \sigma$, is displayed as a function of $\delta$. It is clearly seen that the target efficiency decreases towards smaller coupling strengths. This is a result of the fact that, without coupling, halo particles with $\sigma_{x}>\sigma_{y}$ have little chance to be absorbed in the target before hitting the horizontal aperture limit.

\section{Transient Behaviour of Rates}

While the steady state rates give insight into the target efficiency, the transient behaviour observed after movements of the target can be used to obtain information about the dynamics of the beam halo. In this section we interpret the response of the trigger rates to target movements towards the beam or away from the beam in the framework of the diffusion model, described in section 4.3 .

The rate development after a retraction of the target is most sensitive to details of the diffusion processes. In this case, the rate first drops to a background level and then slowly grows again. while the halo is expanding outwards. An example. taken from the scan in Fig. 6 , is shown in Fig. 16a. The target was operated at a distance of $\approx 8 \sigma$ from the beam core and retracted by $\approx 0.3 \sigma$. Directly after the target movement, the rate increases quite steeply for about $50 \mathrm{~s}$, at which point the slope changes abruptly to a smaller value. This kink in the rate development was also observed in other trials and is not due to a change in beam conditions. 

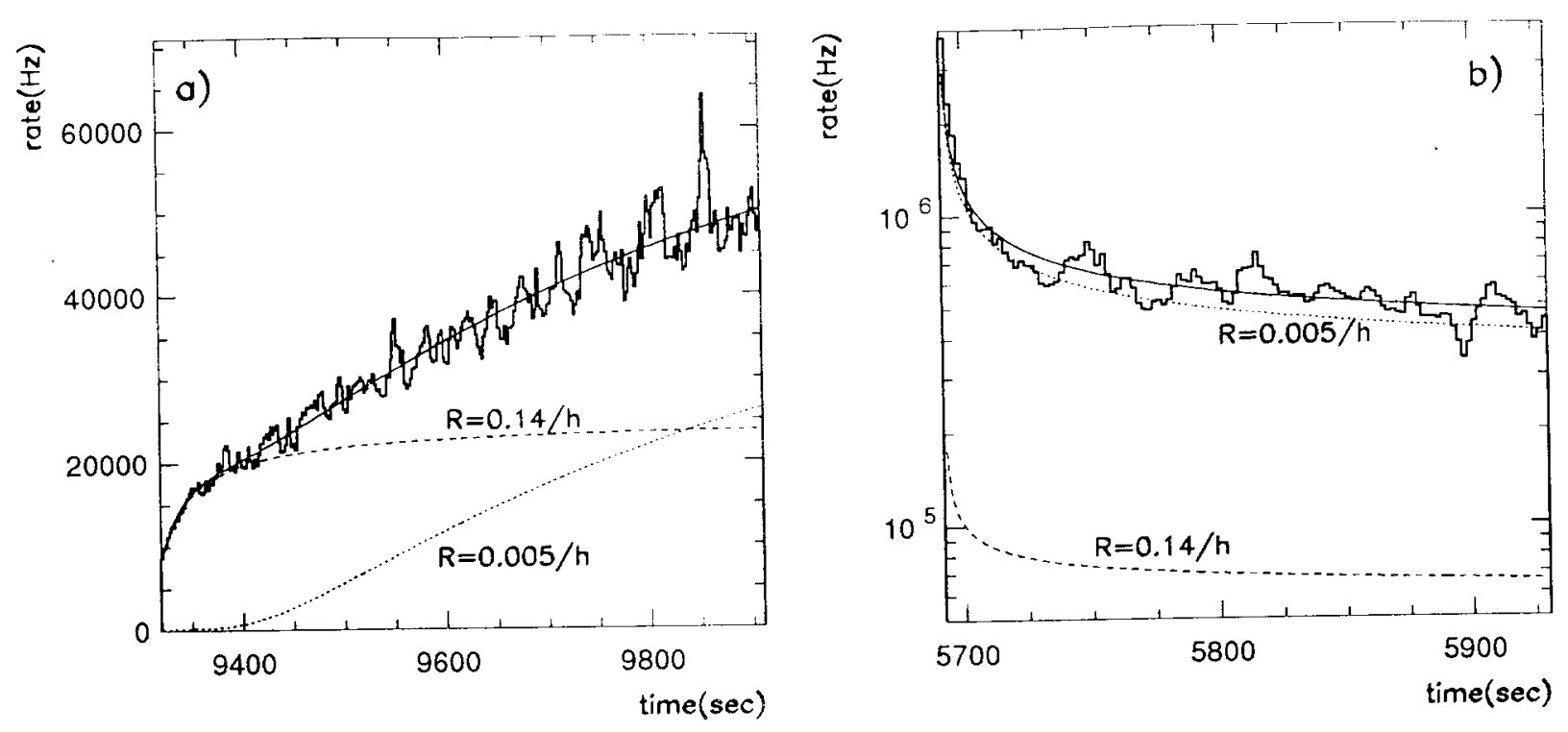

Figure 16: Rate development a) after a retraction of the target by $\approx 0.3 \sigma$ and $b$ ) after a target movement of $\approx 0.15 \sigma$ towards the beam. The data stem from the scan in Fig. 6 . The full curve shows the fit of the diffusion model, the dashed curves denote the contributions of two components with different diffusion constants (see text).

In the framework of the diffusion model, the first $50 \mathrm{~s}$ after the target retraction can be very well described with a diffusion constant $R_{\text {fast }} \approx 0.14 \mathrm{~h}^{-1}$, corresponding to an asymptotic lifetime $\tau_{\text {fast }}=4 /\left(\lambda_{0}^{2} R_{\text {fast }}\right) \approx 5 \mathrm{~h}$. The fit is shown as a dashed line in Fig. 16a. For $\Delta t>50 \mathrm{~s}$ the extrapolated rate from the fit saturates and falls below the data. This long-term increase of the rate can be again described very well by the diffusion model, if a second component of the halo with a smaller diffusion speed is hypothesized. The fit, also shown as a dashed line in Fig. $16 \mathrm{a}$, yields $R_{\text {slow }} \approx 0.005 \mathrm{~h}^{-1}$, corresponding to an asymptotic lifetime of $\tau_{\text {slow }} \approx 140 \mathrm{~h}$ (which is of the same order of magnitude as the overall beam lifetime). At large times (towards the new equilibrium rate of several $100 \mathrm{kHz}$ ) the trigger rate is totally dominated by this slow component. The sum of the two contributions is represented by the full line in Fig. 16a, and is in perfect agreement with the measurements over the full time interval.

The second case, i.e. a target step towards the beam, is shown in Fig. 16b. The target is moved by about $0.15 \sigma$ in this case. After the movement, the rate increases from a few $100 \mathrm{kHz}$ to several $\mathrm{MHz}$ and then decays rather quickly. Qualitatively, this behaviour is explained as follows: in the first second or so, the halo extending beyond the new position of the inner edge of the target is cleaned out. After that, however, the particle density right inside this position is still well above the equilibrium density, and particles continue to diffuse into the range shadowed by the target ribbon at a high rate. Only after much longer times the particle density and hence the rate have reached a new equilibium, described by the asymptotic terms in the solution of the diffusion equation. Due to the high counting rate, one does not expect to be sensitive to a small contribution of the fast component of the halo. The rates should be almost entirely explainable by the slow component alone. The full curve in Fig. 16b represents the superposition of fast and slow component as predicted by the diffusion model with parameters fixed to those obtained from the fits to Fig. 16a. 
The dashed curves indicates the individual contributions of the fast and slow components. As expected, the rate from the fast component is much smaller than that from the slow component. Apart from the first few seconds, where the observed rates might be affected by the clean-up of the halo beyond the inner edge of the target ribbon. the agreement with the data is good within the fluctuations of the measured rates (most probably caused by slight changes in machine conditions).

In summary, it is possible to explain the observed transient behaviours consistently with a single set of diffusion constants. The lifetimes obtained from these diffusion constants are, however. in the order of many hours. This is in drastic disagreement with the fact that the tracking model requires quite large drift velocities $(\approx 20 \sigma / \mathrm{s}$ at $10 \sigma$ and $\approx 0.1 \sigma / \mathrm{s}$ at $8 \sigma)$ in order to explain the finite target efficiencies. These drift velocities lead to lifetimes of the order of a second. At lifetimes of several hours, the target efficiencies would rise to unity very steeply when the target moves out of the collimator shadow. This contradiction between the two aspects of halo dynamics can only be due to the drastic simplifications of the halo models.

One might speculate that the discrepancy could be due to the fact that the halo phase space consists of a mixture of stable, quasi-stable and chaotic regimes. The density of chaotic regions increases steeply with increasing betatron amplitude. Particles which enter chaotic phase space regions move on erratic trajectories and will be lost abruptly, without performing a continuous drift towards large amplitudes. Such particles are probably not absorbed by the target with high efficiency. The slow moving components, however, will be totally absorbed and are responsible for the observed interaction rates. If transient behaviour is analyzed, one is therefore blind to the chaotic parts of phase space, and measures exclusively diffusion constants for stable or quasi-stable regions of phase space. The resulting time constants should hence be comparable to the beam lifetime. If target efficiency is considered, on the other hand, the full loss of beam current is taken into account, irrespectively of its origin. The finite target efficiency would thus be a measure of the fractional volume of chaotic phase space regions inside the boundary given by the target position. Consequently, the apparent drift velocity would be an average of that of the diffusing halo and the effective drift velocity of chaotic trajectories. With decreasing distance of target and beam, the fraction of chaotic regions, not shadowed by the wire, decreases and the target efficiency increases.

A more quantitative interpretation of the observations would require a highly involved analysis of the beam phase space structure, and is certainly far beyond the scope of this paper. We would like to stress, however, that apparently the target rate measurements are able to reveal quit subtle details about the structure of the beam halo at HERA, and may give important experimental input to beam dynamics calculations.

\section{Event Topology}

Without a sophisticated detector it is hard to gain detailed information on the structure of the events produced in the target interactions. Some coarse features can, however, be measured using ADC pulse heights and TDC times for the scintillators.

A measure of the particle density distribution in single interactions can be obtained from the fractions $f_{k}$ of $k$-fold coincidences of the four hodoscopes. We use events taken at. 
relatively modest trigger rates. to avoid the effect of multiple interactions in a single BX. The results are listed in table 3 together with those obtained for the case of background erents taken when the targets were retracted. The coincidence pattern of target events is

Table 3: Fractions. $f_{k}$. of k-fold coincidences of the four hodoscopes for single interactions together with the predictions of event generators combined with detector simulation. Also shown are the same fractions $f_{k}^{B G}$ for background events (see text).

\begin{tabular}{|c|c|c||c|c|}
\hline$k$ & $f_{k}$ measured & $f_{k}$ predicted & $f_{k}^{B G}$ measured & $f_{k}^{B G}$ predicted \\
\hline 0 & 0.41 & 0.41 & 0.52 & 0.53 \\
1 & 0.24 & 0.28 & 0.18 & 0.20 \\
2 & 0.17 & 0.18 & 0.12 & 0.10 \\
3 & 0.12 & 0.10 & 0.09 & 0.08 \\
4 & 0.06 & 0.03 & 0.09 & 0.09 \\
\hline
\end{tabular}

distinctly different from the pattern for background events. The background trigger - caused by upstream interactions and showers - are characterized by higher particle densities and an increased rate of three- and fourfold coincidences.

Also listed in table 3 are the predictions obtained from simulated proton nucleon interactions, using the FRITIOF event generator [14], tracked through the test setup using the GEANT [15] package for the detector simulation. For the case of background events, protons were assumed to interact anywhere in the beam pipe material up to $10 \mathrm{~m}$ upstream of the target position. The agreement between the simulations and the measurements is good. Only the fraction of fourfold coincidences from interactions in the target is somewhat too small in the predictions. The most probable reason is the fact that the rather complicated and very massive mounting of the detector as well as the beam line components (none of which have been optimized for minimal total radiation length) are not taken into account in full detail in the simulations and therefore the number of secondary interactions is underestimated. Since the same simulation was used to estimate the acceptance of the trigger counters for target interactions, the result of $\approx 95 \%$ for this quantity has to be regarded as a lower limit.

Single and multiple coincidences of scintillator hodoscopes correspond on the average to different particle multiplicities produced in the interactions in the target ribbon. The particle multiplicity, on the other hand, is reflected by the ADC spectra of the trigger counters. Fig. 17 displays the ADC spectra of the trigger counters for events in which only one hodoscope was fired and for events in which all four hodoscopes fired simultaneously. The spectra clearly demonstrate that the fourfold coincidences correspond on the average to much higher multiplicities, possibly connected with multiple interactions per BX.

Target interactions and background can be very clearly distinguished when analyzing the particle density in the backward hemisphere: Fig. 18 displays the ADC spectra obtained from the VETO counters for the case of retracted target (background) and for the case of target interactions. The background spectra are very flat and have a large overflow, compared to the pretty narrow spectrum obtained from interactions in the target. This agrees with the expectation that background events are very busy and often consist of big showers hitting the 


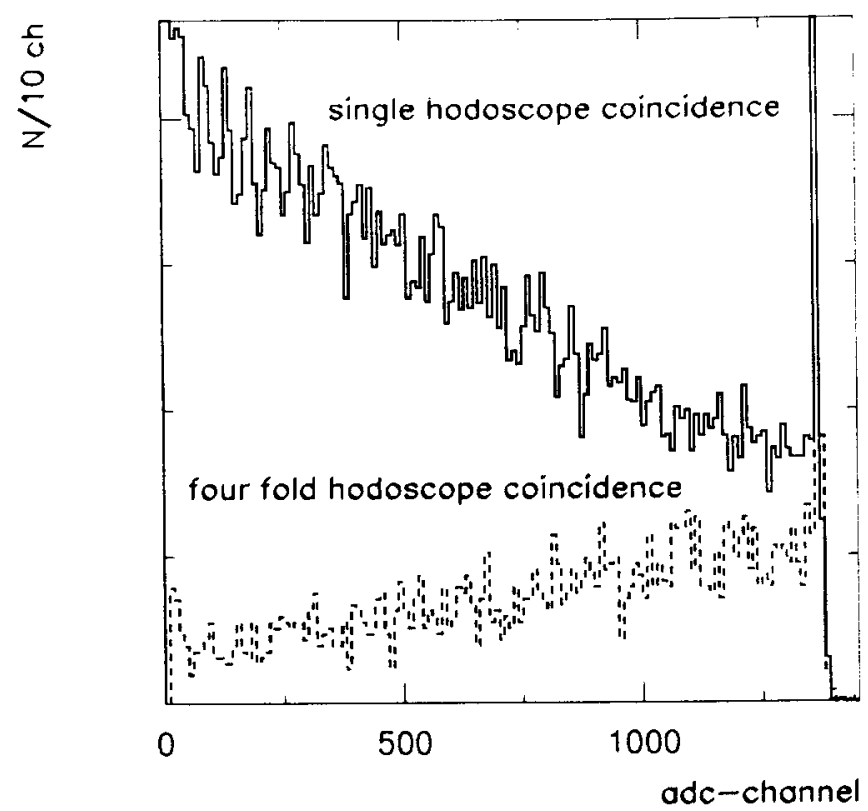

Figure 17: ADC spectra of the trigger counters for target interactions with a single hodoscope fired and with all four hodoscopes fired. The spike at the right edge represents $A D C$ overflows.

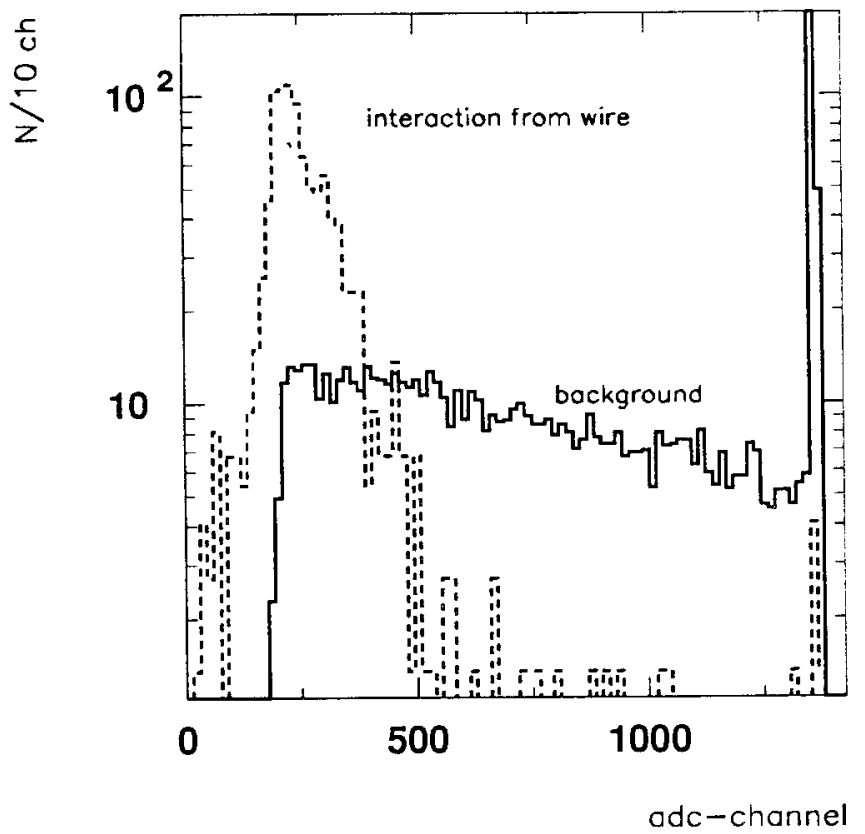

Figure 18: ADC spectra obtained from the VETO counters for the cases of background running and interactions in the target. The spike at the right edge represents ADC overflows. 


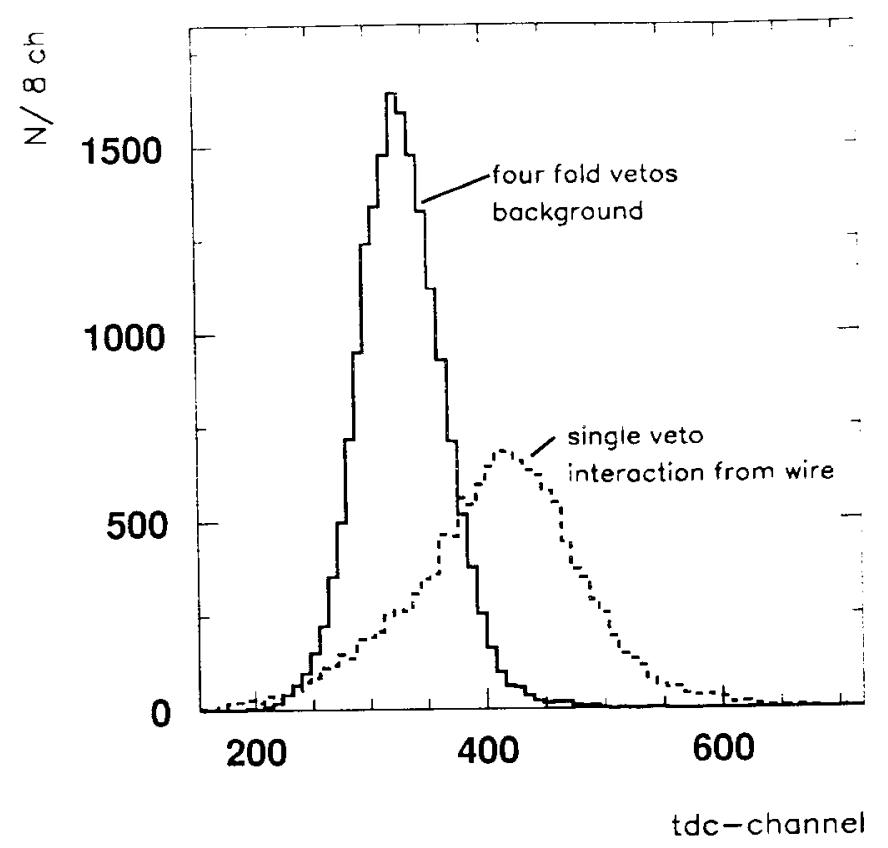

Figure 19: Time delay between the TDC stops of the trigger counters and the VETO counters in the cases of background events with all VETO counters firing and target interactions with a single VETO counter set. One TDC channel corresponds to $0.1 \mathrm{nsec}$.

counters. In target interactions, however, the multiplicity of particles scattered backwards is very small, and VETO counters are mostly hit by single particles.

Backscattered particles can originate in secondary interactions in the material of the detector or the beam guiding system; they can also be due to nuclear fragments, very soft and isotropically emitted particles (mostly protons) from proton nucleus interactions [13]. If the veto is set by particles from upstream background reactions, particles will hit the veto counter about 6 ns earlier than the trigger counters. In contrast, slow fragments or backscattered particles caused by reactions in the wire should reach the veto counters with a time delay. Fig. 19 represents the delay between the TDC stop signals of the trigger counters and the VETO counters for the cases of fourfold VETO events (background running) and target interactions in which a single VETO is set (the absolute time scale is arbitrary since no absolute TDC time calibration has been done). It is indeed observed that the single VETO hits in target interactions are delayed by several ns with respect to background events.

\section{Rate Fluctuations in the Interaction Rates}

Up to now the analysis concentrated on time averaged quantities like mean interaction rates per $\mathrm{BX}$ or trigger rate per second. The performance of a high rate $B$ experiment would, however, suffer from interaction rate fluctuations since the detector can only disentangle a certain maximum number of superimposed interactions in one BX. There are three sources of such fluctuations: 


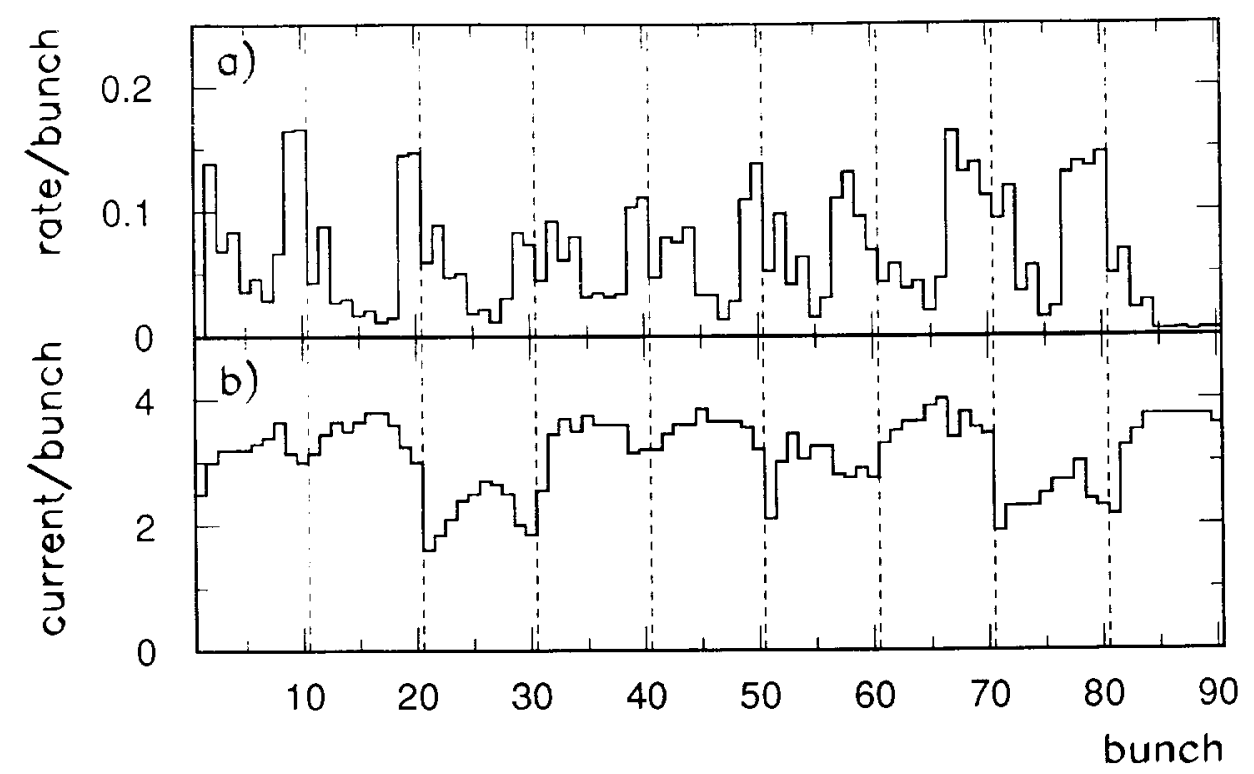

Figure 20: Mean interaction rate (a) and current (b) per bunch as a function of bunch number. The dashed lines indicate the boundaries between consecutive bunch trains.

1. Poissonean fluctuation of the number of interactions per BX in every bunch.

2. Bunch to bunch variations due to non-uniformities in the injection procedure of the storage ring. These include, e.g., variations in bunch currents, variations in the bunch emittances or variations in the orbit from bunch to bunch.

3. Rate variations due to external disturbances of the beam. These include power supply ripples, vibration of the beam guiding system due to motors, pumps etc., as well as ground tremor.

The first, purely stochastic fluctuations can of course not be avoided and will ultimately limit the interaction rate to be recorded by the detector. The second and third source of fluctuations are of external origin and can possibly be avoided to a large extent. They will be discussed in the following sections.

\subsection{Bunch to Bunch Variations}

Bunch to bunch variations of the interaction rates can be studied experimentally using the pulses of the trigger counters as recorded by the Flash-ADC system. Fig. 20a shows the interaction rate per $\mathrm{BX}$ as a function of bunch number for a run where these variations are particularly strong ${ }^{7}$. Note that the last 6 bunches do not collide with the electron beam and do therefore not contribute significantly to target interactions since their halo is not well populated. For the other bunches the interaction rates vary by an order of magnitude. In addition, a systematic structure within the bunch trains of ten consecutive bunches can be

\footnotetext{
${ }^{7}$ Since bunch 1 is the trigger bunch and is therefore biased to always leave pulses in the Flash-ADC spectra, its mean interaction rate camnot be determined in this way.
} 


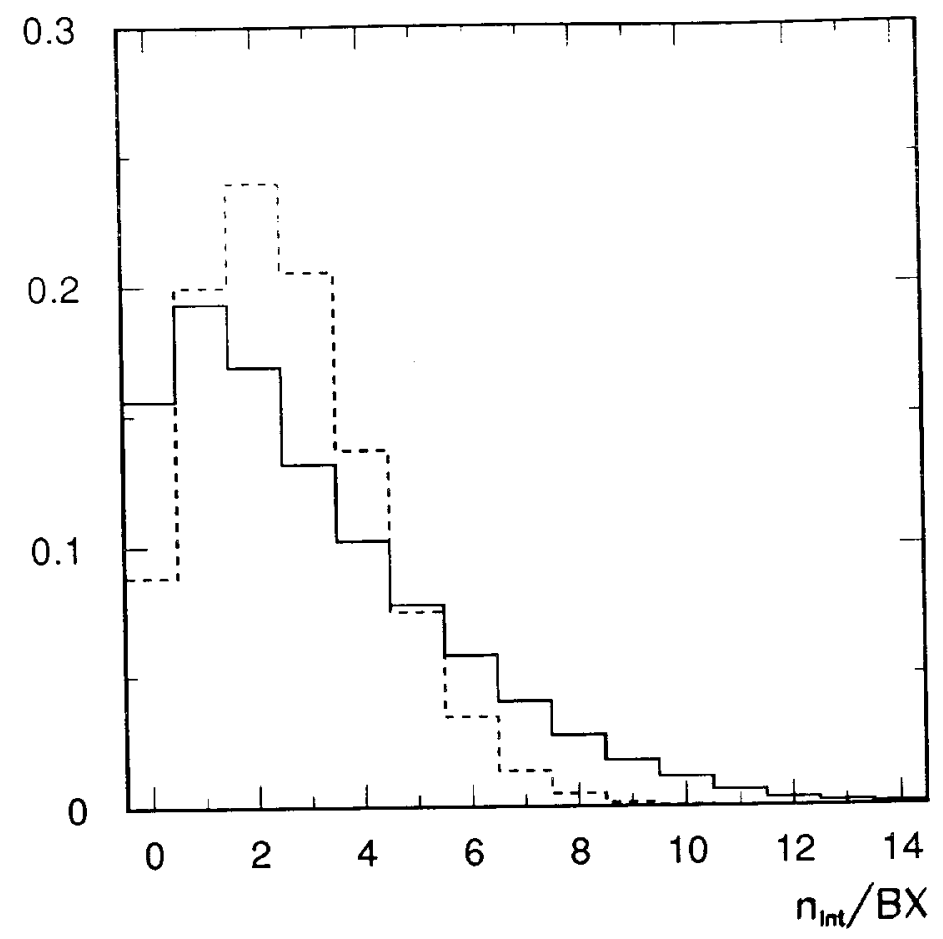

Figure 21: Distribution of the number of interactions per BX for the situation in Fig. 20, scaled to an average interaction rate of $3 / B X$ (full histogram). The dashed histogram represents a Poisson distribution with mean value 3.

recognized. This clearly points to a systematic problem connected to the injection procedure, like de-adjusted kicker timing or insufficient lengths of the kicker flat top phases.

Fig. 20b displays the bunch current (arbitrary units) for the same run, as measured from the pulse heights of the beam pick-up signals, as a function of bunch number. Although two bunch trains are systematically low in current by $50 \%$, the overall current variations are very small as compared to the observed variations in interaction rate. This indicates that the rate variations are mainly due to significant bunch to bunch variation of emittances.

In the run shown in Fig. 20, the bunch to bunch r.m.s. variations of the interaction rate is $66 \%$, the variation in the current is $20 \%$. On average, fluctuations were smaller, about $10 . .20 \%$ in the bunch rate and $10 \ldots 20 \%$ in the bunch currents.

In Fig. 21 the consequences of these quite extreme bunch to bunch variations are demonstrated for an experiment operated at an average interaction rate of $3 / \mathrm{BX}$. The full histogram represents the distribution of the number of interactions per $\mathrm{BX}$, if the situation shown in Fig. 20a is scaled to $\left\langle n_{I n t}\right\rangle=3$. Superimposed is the Poisson distribution with mean value 3 , expected if no variations were present. If the detector could handle up to 6 superimposed interactions. about $11 \%$ of all events ( $30 \%$ of all interactions) would be lost. In the case of the typical observed bunch to bunch variations, the corresponding number is $3.6 \ldots 4.2 \%$ $(9.0 \ldots 10.7 \%)$ and for the purely poissonean fluctuation $3.4 \%(8.4 \%)$.

In conclusion. the observed bunch to bunch variations can in some extreme cases affect the performance of a high rate experiment. It is important that the injection procedure is carefully tuned and is to a large extent reproducible in quality. It should be stressed that 

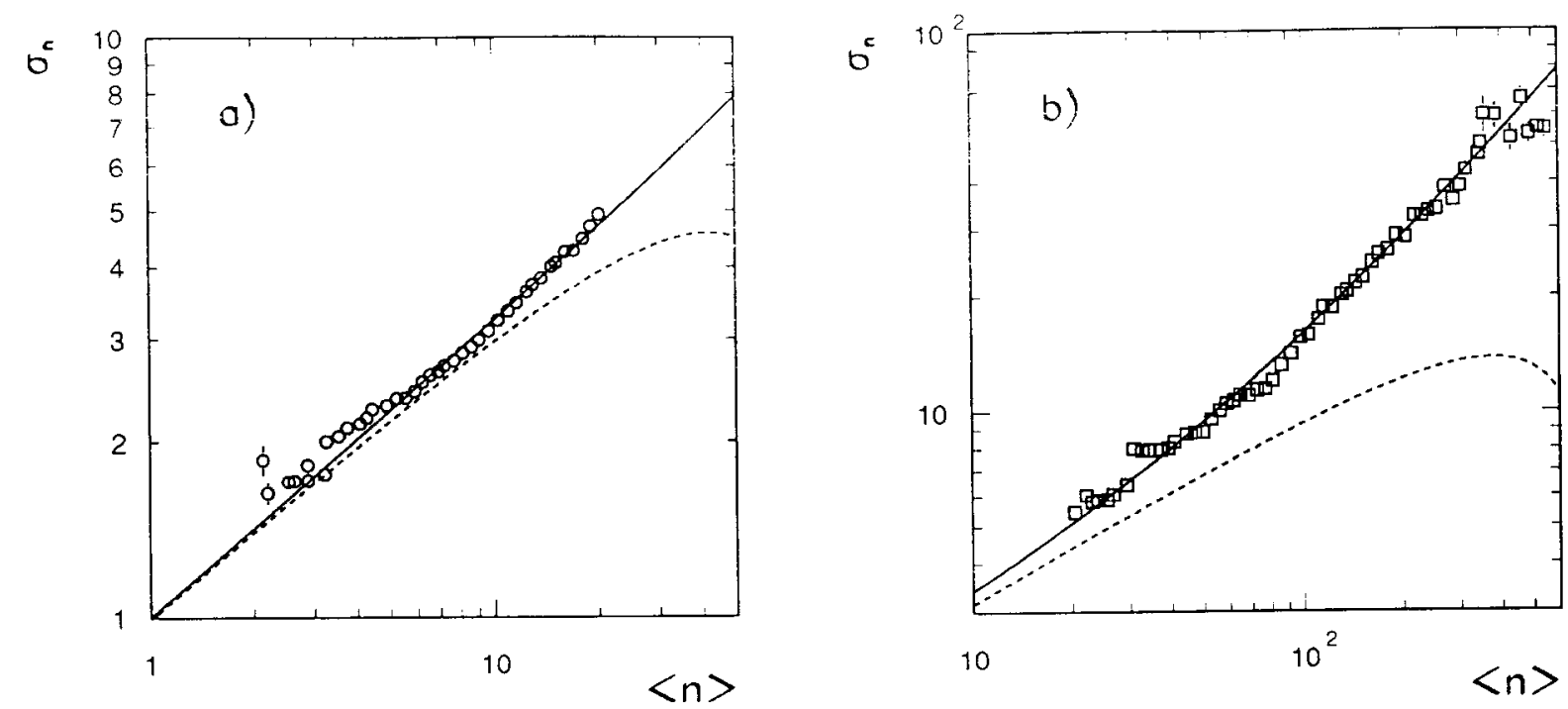

Figure 22: R.m.s. variation of the number of triggers per beam revolution (a) and the number of triggers per nine beam revolutions (b) as a function of the corresponding mean value. The full lines represent the model using a convolution of the ideally expected binomial distribution for $84(9 \times 84)$ bunches with a Gaussian of a relative width of $13 \%$ (see text); the dashed lines are the same prediction without convolution with a Gaussian.

the above example was the most extreme case observed. In typical runs the bunch to bunch variations were small enough $(10 \ldots 20 \%)$ to be acceptable.

\subsection{Rate Modulation}

In order to provide a measure of longer term modulations in the interaction rates, the number of triggers was repeatedly counted for a time of one beam revolution and for a time of nine beam revolutions during a target scan. We denote by $N$ the total number of bunches counted ( 84 or $756=9 \times 84$ ), and by $n$ the number of triggers from these bunches. The mean trigger probability is $p=\langle n\rangle / N$. For purely statistical rate variations, the probability to observe $n$ triggers is described by a binomial distribution $b(n, N)$, with an r.m.s. width

$$
\sigma(n)=\sqrt{N \cdot p \cdot(1-p)}=\sqrt{\langle n\rangle} \cdot \sqrt{1-\frac{\langle n\rangle}{N}} .
$$

In Fig. 22 the observed r.m.s. fluctuation of the trigger rate $n$ is plotted as a function of its mean value $\langle n\rangle$. The dashed line shows the expected width of the binomial distribution. For small values of $\langle n\rangle$ data are consistent with the expectation; at larger values of $\langle n\rangle$ the fluctuations are larger than the predictions, and $\sigma(n)$ is proportional to $\langle n\rangle$ rather than to $\sqrt{\langle n\rangle}$. Data are well described over the full range. if the binomial distribution is convoluted with a Gaussian of relative width of $13 \%$, as can be seen from the full lines in Fig. 22 .

Obviously, beam parameters relevant for the interaction rate fluctuate on time scales long compared to the time needed for 9 revolutions, or $180 \mu \mathrm{s}$, equivalent to frequencies in the $\mathrm{kHz}$ range or below. This external noise component of $13 \%$ is equivalent to a beam position jitter of $10-20 \mu \mathrm{m}$ (or jitter in any other beam parameter which affects the trigger rate, like 

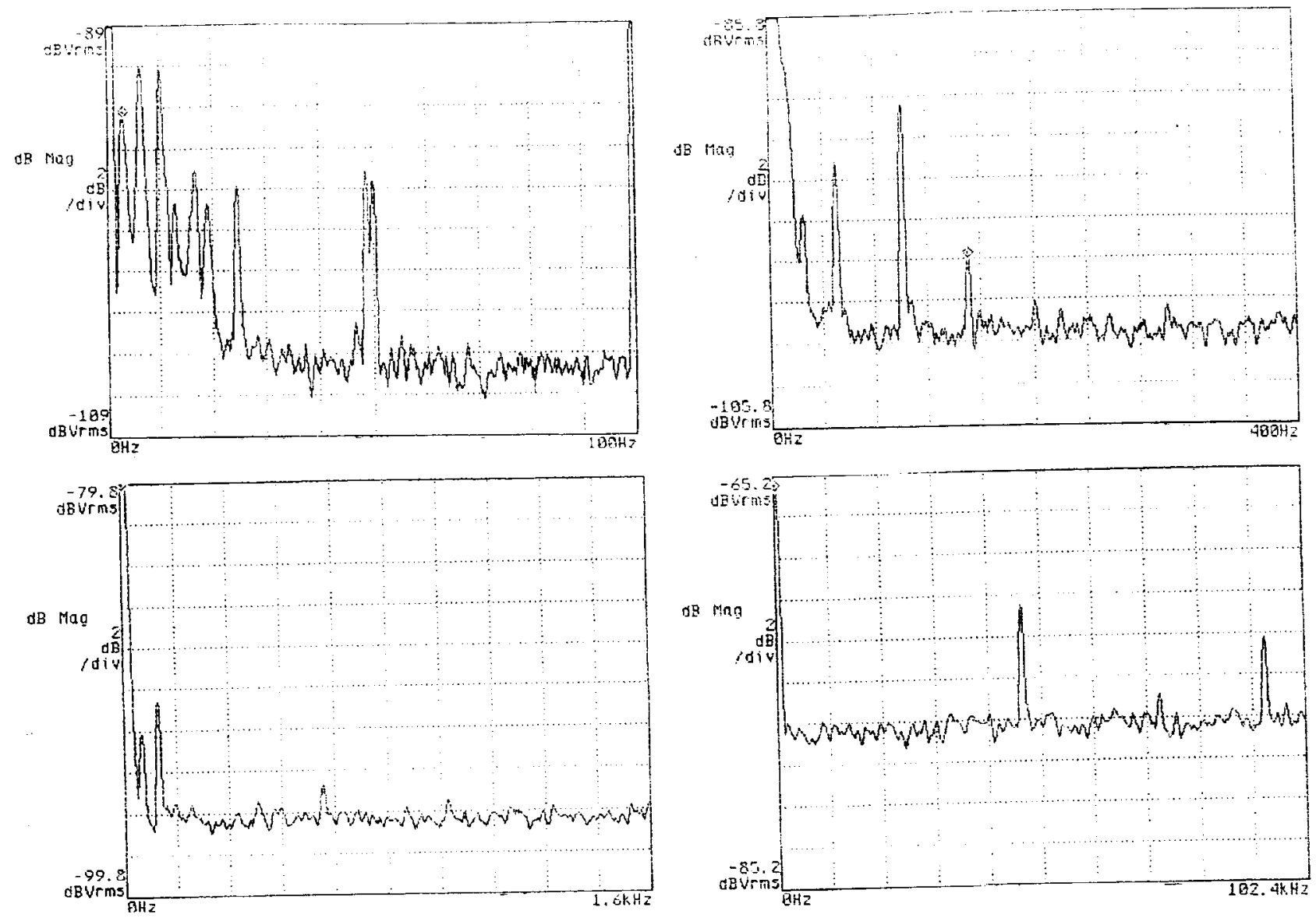

Figure 23: Frequency spectra of the trigger signal derived from the test experiment.

beam emittance). This is a small fraction of the r.m.s. beam width (see table 1). Although modulations in the trigger rate are visible, their magnitude is not large enough to affect a $B$ experiment significantly.

It is nevertheless interesting to search for the sources of rate modulations in order to understand whether they might be avoided in the future. Fig. 23 shows frequency scans recorded by a frequency analyzer which was fed with the trigger signal of the test experiment. Clearly seen are the harmonics of the revolution frequency $(47 \mathrm{kHz})$, but also side bands at very low frequencies, at $50 \mathrm{~Hz}$ and a structure around $600 \mathrm{~Hz}$. The latter two frequencies are characteristic for the power supplies used at HERA. The structures at lower frequencies are more likely to be connected to mechanical vibration resonances in the beam guiding system. Similar modulations have been observed in other machine related quantities like the tunes, orbits and the loss rates on collimators [16].

\section{Rate Control}

One important aspect of the operation of a high rate $B$ experiment is the continuous position control of several halo targets surrounding the beam, in order to guarantee that the interaction rates stay constant and are equally distributed over all wires. This goal can in principle be achieved by two feedback loops which act on quantities which are essentially 
orthogonal:

1. Absolute rate feedback: This feedback loop is fed by the total trigger rate and it reacts by moving simultaneously all target wires inwards or outwards. In first approximation. this does not change the relative rates from target to target.

2. Relative rate feedback: This feedback loop is fed by the individual interaction rates from the different target wires. This can for example be achieved using online event reconstruction in the DAQ chain of the experiment. Less sophisticated methods are discussed below. The feedback then moves targets with high relative rates outwards and those with low relative rates inwards, such that the total rate is in first approximation unaffected.

The absolute rate feedback was implemented and tested during the 1993 running period. The target was in this case operated directly by the main control system of the HERA proton ring which was fed with trigger rates and target positions by a dedicated front end control PC in the test experiment counting area. During a half hour test, the trigger rate was successfully stabilized on a pre-set value of $1 \mathrm{MHz}$. No instability in the feedback system was observed.

The relative rate feedback is much more complicated and could not be tested in practice up to now. It is likely that the position of online reconstructed vertices will be the input to the feedback in a large and sophisticated $B$ experiment. There are, however, also much simpler tools which are probably not as accurate but possibly usable already in the present. test experiment.

1. It is conceivable that the target temperature or an electric current drawn by the target are a good measure of the rate of protons crossing the target material. These ideas would require modification of the target mounting and can not be tested at the moment.

2. When the target is moved, its transverse distances to the scintillators of the hodoscopes change. This has two effects which are expected to be of similar magnitude (from FRITIOF simulations): With increasing distance to a target ribbon, the solid angle covered by a scintillator hodoscope decreases. At the same time, the particle flux through the scintillator decreases due to the steep polar angle distribution of particles generated in the interactions. The relative change of the hodoscope rate is expected to be of the order of $1 \%$ for a $1 \mathrm{~mm}$ target movement. Such effects were indeed observed very cleanly. Fig. 24 displays the left-right and up-down asymmetries

$$
A_{L R}=\frac{R_{L}-R_{R}}{R_{L}+R_{R}} \quad A_{U D}=\frac{R_{U}-R_{D}}{R_{U}+R_{D}}
$$

for events in which at most three hodoscopes fired (events with fourfold coincidences do not contribute to a rate asymmetry). The quantities $R_{L}, R_{R}, R_{U}$ and $R_{D}$ denote the rates measured with the left, right, upper and lower hodoscope. The asymmetries are displayed for individual scans of the vertical and the horizontal $\mathrm{Cu}$ ribbon. As expected, the left-right asymmetry changes during the scan of the vertical target and the up-down asymmetry during the scan of the horizontal target. For the other two 

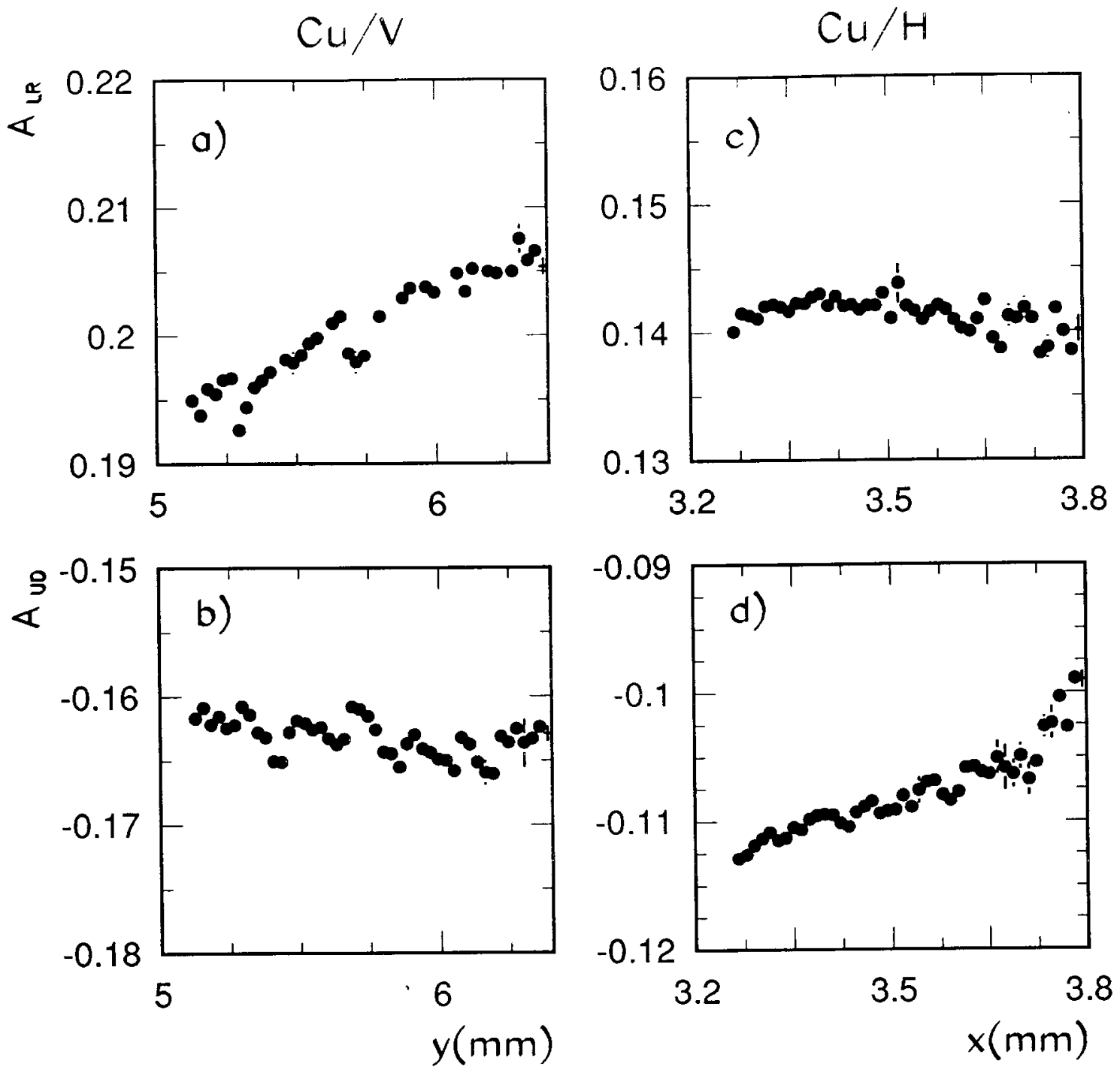

Figure 24: Left-right $(a, c)$ and up-down $(b, d)$ asymmetries of the hodoscope rates measured in scans of the vertical $\mathrm{Cu}$ target $(a, b)$ and the horizontal $C u$ target $(c, d)$.

combinations the influence of the target position is smaller (the residual effect is due to the fact that the hodoscopes are not exactly oriented in left, right, upwards and downwards directions). For a target movement of $1 \mathrm{~mm}$ the relevant asymmetries change by $1 \%-2 \%$ which agrees with the expectations.

This observation can be exploited for a simple but effective relative rate control. Fig. 25 shows a lego plot of the distribution of the two asymmetries measured during the time of a series of scans of the horizontal and vertical $\mathrm{Cu}$ target. Clearly seen are two well separated peaks in the $A_{L R}, A_{U D}$ space corresponding to situations where only one of the two targets is positioned in the halo. The third peak in between represents the situation where both targets are moved close to the beam and equally sharing interactions. It is obvious that this opens sensitive and simple tools for automatic control of the relative target rates. 


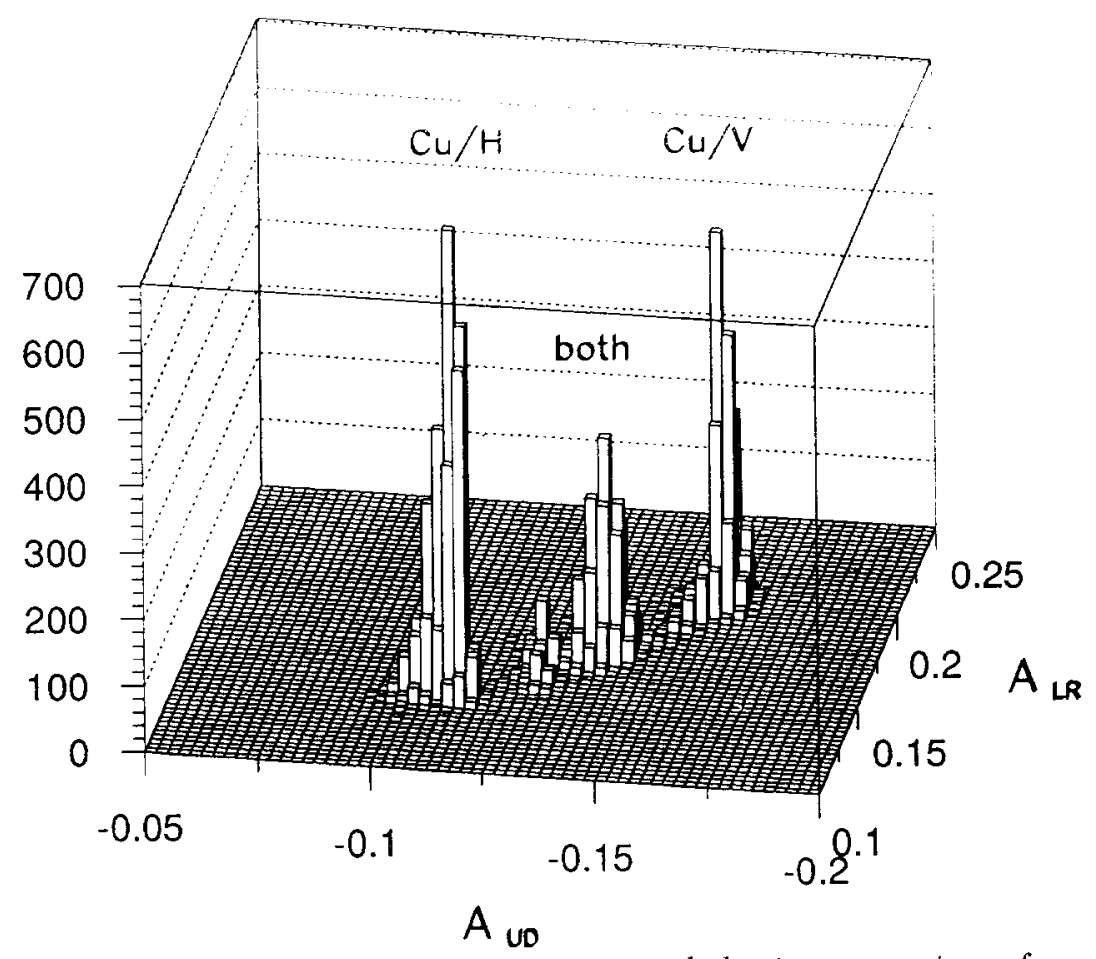

Figure 25: Distribution of rate asymmetries measured during a series of scans of the horizontal and vertical Cu target.

\section{Compatibility with ep Operation}

We have shown above that very high interaction rates are achievable with internal targets in the HERA proton ring. A successful operation of a high rate target requires in addition that neither the smooth machine operation is jeopardized nor any excessive backgrounds are produced which could deteriorate the performance of other HERA experiments.

As far as the machine operation is concerned, no negative effect of the presence of the internal target was noticed. In particular, the target did not affect the beam orbit, the emittances or the tunes. The only exception is the lifetime of the beam which is affected after every target movement. This change of lifetime is dominated by the halo lifetime and does not concern the lifetime of the beam core, i.e. the luminosity lifetime. If, e.g., the target moves towards the beam, some halo is scraped away and the apparent beam lifetime decreases. As soon as a new equilibrium beam phase space population is reached, the lifetime has normally recovered to roughly its old value. If the target is moved away from the beam, the opposite phenomenon is observed: Until the halo (which starts to expand again) arrives back at the target, the apparent beam lifetime becomes very large and finally settles back around the old value.

It should be noted that in several occasions the natural lifetime of the proton beam was observed to be extremely large (in the order of several hundred hours). If this is the case even with the machine running at design currents. the necessary interaction rate of a $B$ experiment can only be achieved by running the target sufficiently close to the beam such that the lifetime decreases to an equilibrium value of $100 \mathrm{~h}$. This could introduce a minor 
loss of luminosity for the ep experiments.

Concerning the interference with other experiments three aspects should be mentioned:

- Background to the ep detectors: The backgrounds of the ep experiments were monitored during all test runs and were never drastically increased by the target. As an example, Fig. 26 displays the ZEUS background counting rate [17] observed during a target scan with wire steps of $30 \mu \mathrm{m}$ and trigger rates of up to $1 \mathrm{MHz}$. The tar-

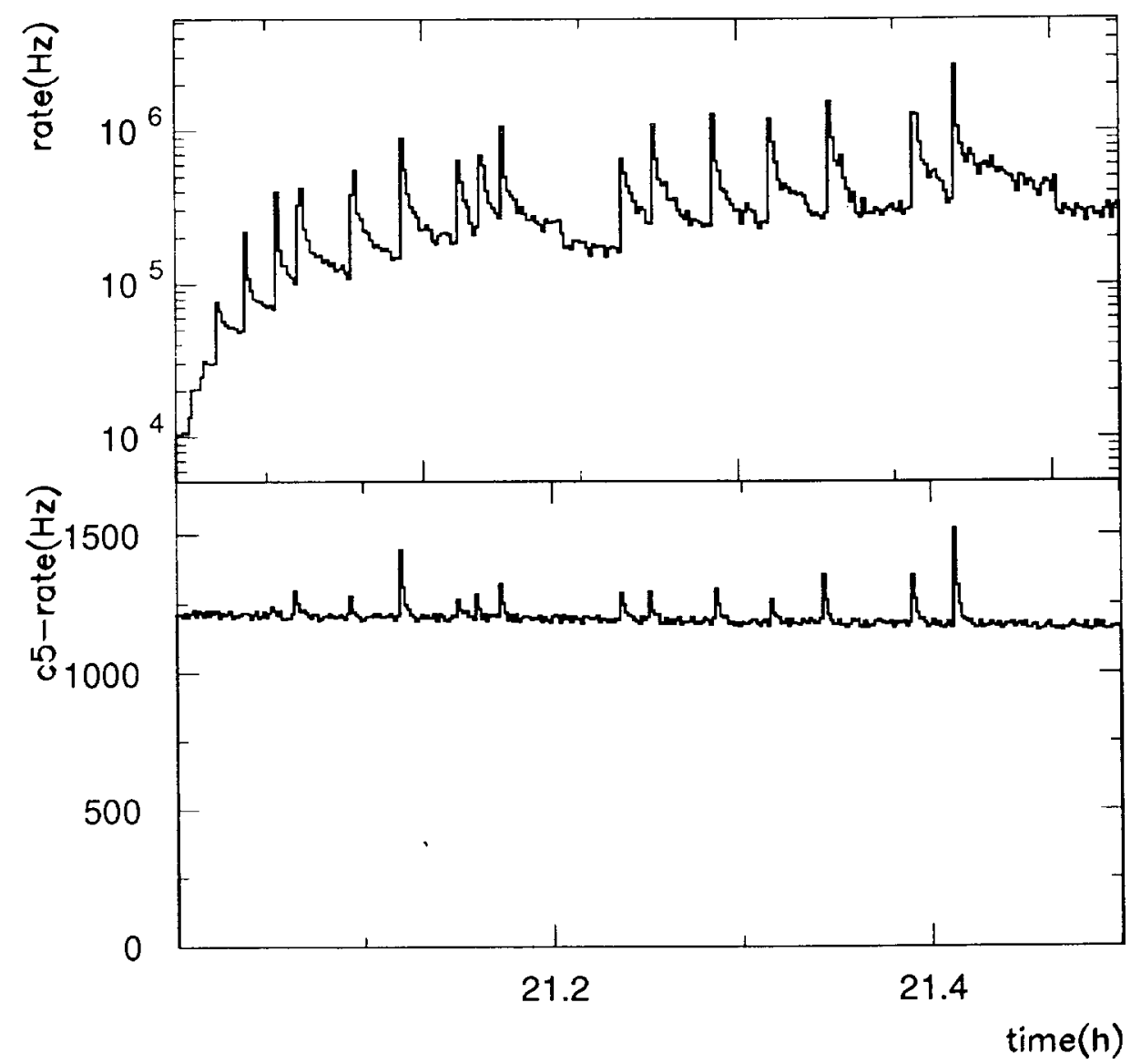

Figure 26: Background counting rates observed by the ZEUS background detectors during a target scan with wire steps of $30 \mu \mathrm{m}$ and trigger rates of up to $1 \mathrm{MHz}$.

get movements can be noticed as small spikes in the background rate. No long term deterioration of running conditions can be seen.

- Neutral Background along the beam axis: The main detectors are blind to such a background component since neutral particles are not deflected into the detector. However, a neutron calorimeter, positioned behind the first dipole magnets $(100 \mathrm{~m}$ downstream of the ZEUS detector) and covering the acceptance of $0^{\circ}$ neutrals, was commissioned during the last running period [17]. The background in this instrument turned out to be highly sensitive to smallest variations of beam conditions and showed amongst other sources a clear sensitivity to the internal target. It still has to be clarified whether the observed background might be prohibitive for routine running [17]. 
- Forward detectors in the vicinity of the beam. The ep experiments are now operating forward detectors which are mounted inside "roman pots" and can be moved close to the beam. Clearly, in routine running, these detectors should be protected from beam losses and should be shadowed by the main collimators (typically positioned at $10 \sigma$ ). If, however, due to misalignment of detectors or mis-steering of the beam orbit the detectors themselves become aperture limiting (and hence unprotected), they start to compete with the internal target in absorbing the beam halo. Such an incidence was observed once during the commissioning phase of the ZEUS forward detectors in 1993. Due to orbit mis-steering the detectors moved in to a distance of about 5 r.m.s. beam widths and totally shadowed the internal target. The counting rate dropped from the $\mathrm{MHz}$ range to a value smaller than the normal background rate and recovered immediately when the ZEUS detectors were moved out again. In order to allow the running of a high rate experiment with an internal target, it is therefore important to carefully control all other detector components which are operated in the vicinity of the beam and verify that they are at all times well shadowed by the protecting main collimators.

\section{Summary and Outlook}

The feasibility of utilizing internal wire or ribbon targets in the halo of the HERA proton ring has been demonstrated. With less than $10 \%$ of the design current stored in the machine and less than $20 \%$ of the design current per bunch, up to $8 \mathrm{MHz}$ of interaction rate and 2 interactions per bunch crossing have been observed. Target interactions are clearly distinguished from background events in their event topology, in the timing information of the scintillation counters and in the distribution of track directions reconstructed in the drift chambers. Already at distances from the beam of about $8 \sigma$, target efficiencies in excess of the goal of $50 \%$ have been routinely achieved. No excessive backgrounds (possibly with the exception of background to neutron calorimeters at $0^{\circ}$ ) have been observed and neither orbit nor tune or emittance of the proton beam were affected by the target.

The target efficiency has been studied in simple single particle tracking models, simulating the linear machine, the halo diffusion, and scattering in the target material. Within the uncertainties of the exact structure of the machine aperture, the observed dependences of target efficiencies on the target positions can be qualitatively understood. The models indicate that the aperture, defined by the main collimators, as well as the $\beta$ function at the target position are the decisive factors for the magnitude of the target efficiency.

The halo particle dynamics can be studied by analyzing the transient behaviour of event rates after target movements. The transients could be explained in the framework of diffusion models where the lifetimes of the diffusing halo components are comparable to the beam lifetimes. This observation is in contradiction to the interpretation of target efficiency within single particle tracking models, based on diffusion-like processes. We conclude that only part of the halo is subject to pure diffusion. The rest might be identified with chaotic regions where particles move on erratic orbits and can be lost abruptly.

The observed event topology shows evidence for very slow particles, possibly nuclear fragments or particles from secondary interactions, scattered backwards from the target 
(or surrounding material of the detector or the beam guiding system). The relative $k$-fold coincidence rates of scintillator hodoscopes were measured and compared to standard event generators, combined with detector simulations. The agreement between the simulations and the data was found to be reasonable. given the limited level of detail at which the detector environment could be implemented in the simulation programs.

Rate fluctuations due to bunch to bunch rate variations. most probably due to different halo emittances of the bunches. were observed in part of the test runs. These fluctuations can be big enough to deteriorate the performance of a high rate $B$ experiment. Care has to be taken in tuning and stabilizing the injection procedures in order to avoid such effects.

Rate modulations due to external sources were observed to increase the intrinsic stochastic rate fluctuations by a very small amount. They are unlikely to influence the performance of a $B$ experiment.

The total target rate has been successfully controlled by an automatic feedback loop. Evidence has been presented that relative rate control might be possible by using asymmetries between trigger rates of the different scintillator hodoscopes.

Finally. many of the described measurements of the relatively simple test experiment showed remarkable sensitivity to details of the machine set-up, like differences in emittances and currents from bunch to bunch, external sources of beam excitation or tiny orbit movements. As a consequence, the internal target set-up might well serve as a valuable and sensitive tool for machine diagnostics purposes.

The test experiment has been upgraded for the 1994 HERA running. The trigger scintillators were replaced by 24 small scintillators, covering the same area. This will reveal much more details on the event topology and will avoid excessive rates in single counters. Likewise, the scintillators in the hodoscopes were replaced by smaller scintillators in order to decrease the solid angle acceptance of the hodoscopes and therefore increase their high-rate capabilities. The tracking chambers are replaced by new drift chambers with smaller cell size. Non movable "roman pots" have been installed close to the target and will be equipped with several layers of a silicon strip detector. This is expected to increase the resolution of track direction measurements drastically and will allow to associate tracks and targets on an event by event basis. Even vertex distributions along the target and hence the measurement of particle density distributions in the beam halo might come within reach.

\section{Acknowledgments}

The target test measurements would not have been possible without the support from the Machine Group. In particular, we thank F. Willeke for many profound discussions and for his enthusiastic support, also concerning the interfacing between the experiment and the machine. M. Seidel has shared with us his understanding of the HERA collimator system and of the unfathomable physics of the beam halo.

We would like to thank E. Michel for doing all the work, R. Wurth for being on time, ZEUS and $\mathrm{H} 1$ collaborations for recognizing that we exist. 
We acknowledge the generous support and encouragement which we received from the DESY directorate, and thank for the hospitality extended to the visiting groups.

C. Hast was supported by the Bundesministerium für Forschung und Technologie under contract number 0.54D051P. 


\section{References}

[1] J.H. Christenson. J.W. Cronin. V.L. Fitch and R. Turlay, Phys. Rev. Lett. 13 (1964) 138.

[2] S.L. Glashow, Nucl. Phys. 22 (1961) 579; A. Salam, in Elementary Particle Theory, ed. N. Svartholm (Almquist and Wiksell, Stockholm, 1968); S. Weinberg, Phys. Rev. Lett. $19(1967) 1264$.

[3] R. Aleksan et al.. (P Tiolation in the B Meson System and Prospects at an Asymmetric $B$ Meson Factory. in Proc of the ECFA Workshop on a European B-Meson Factory, ed. R. Aleksan and A. Ali, ECFA 93-151; and references therein.

[4] KEK Reports 90-23 (1990); 90-24 (1990); 93-1 (1993).

[5] SLAC Report 352 (1989); 353 (1989); 372, 373 (1991).

[6] H. Albrecht et al., An Experiment to Study CP Violation in the B System Using an Internal Target at the HERA Proton Ring, Letter of Intent, DESY-PRC 92/04 (1992). T. Lohse et al. HERA-B. An Experiment to Study CP Violation in the B System Using an Internal Target at the HERA Proton Ring, Proposal, DESY-PRC 94/02 (1994).

[7] F. Hinterberger, T. Mayer-Kuckuk and D. Prasuhn, Nucl. Inst. Meth. A275 (1989) 239; C. Tschalär, Nucl. Inst. Meth. A308 (1991) 471.

[8] H.R. Koch et al., Nucl. Inst. Meth. A271 (1988) 375.

[9] J. Rieling, Diploma Thesis, MPI Heidelberg (1994), unpublished.

[10] M. Reber, Diploma Thesis, MPI Heidelberg (1994), unpublished.

[11] F. Zimmermann. Emittance Growth and Proton Beam Lifetime in HERA. DESY 93059 (1993).

[12] M. Seidel, Determination of Diffusion Rates in the Proton Beam Halo of HERA, DESY HERA 93-04 (1993).

[13] A. Abduzhamilov et al., Z. Phys. C40 (1988) 1, Z. Phys. C40 (1988) 223;

J.A. Gaidos et al., Phys. Rev. Lett. 42 (1979) 82;

J.E. Finn et al., Phys. Rev. Lett. 49 (1982) 1321;

A.S. Hirsch et al.. Phys. Rev. C29 (1984) 508.

[14] B. Andersson. G. Gustafson and Hong Pi, LU-TP-92-20 (1992).

[15] GEANT. Detector Description and Simulation Tool, CERN Program Library, W5013, version 3.16 (1993).

[16] O.S. Brüning, K.-H. Meß, M. Seidel and F. Willeke, Measuring the Effect of an External Tune Modulation on the Particle Diffusion in the Proton Storage Ring of HERA, DESY HERA 94-01 (1994). 
[17] W. Bartel, U. Schneekloth, Backgrounds in H1 and ZEUS from the HERA-B wire target, H1/ZEUS Note 1 (1994). 